\title{
On the (Sequential) Majority Choice of Public Good Size and Location ${ }^{1}$
}

\author{
Philippe De Donder $^{2} \quad$ Michel Le Breton ${ }^{3} \quad$ Eugenio Peluso ${ }^{4}$
}

February 2012

${ }^{1}$ Earlier versions of this work have been presented at the 9th SAET Conference on Current Trends in Economics, Ischia (Italy), the 31st Bosphorus Workshop on Economic Design, Turkey, and the 2009 ADRES Conference in honor of Maurice Salles, Caen (France). We would like to thank participants and a referee for their comments and suggestions.

${ }^{2}$ Toulouse School of Economics (GREMAQ-CNRS and IDEI), Manufacture des Tabacs (MS102), 21 allée de Brienne, 31000 Toulouse, France. Tel: (+33) 5611285 42. Fax $(+33) 561128637$. Email: dedonder@cict.fr

${ }^{3}$ Toulouse School of Economics (GREMAQ and IDEI), France. Email: lebreton@cict.fr.

${ }^{4}$ Department of Economics, University of Verona, Italy. Email: eugenio.peluso@univr.it. 


\begin{abstract}
This paper studies majority voting over the size and location of a public good when voters differ both in income and in their preferences for the public good location. Public good provision is financed either by a lump sum tax or by a proportional income tax. We analyze both the simultaneous and the sequential determinations of the public good's size and location. We show that, while the choice of the type of public good follows the traditional median logic, the majoritarian determination of the taxation rate need not coincide with the preferences of a median income citizen.

With lump sum financing, income heterogeneity plays no role and the sequential equilibrium consists of the median location together with the public good level most-preferred by the individual located at the median distance from the median. This policy bundle also constitutes an equilibrium with simultaneous voting in the special case of a uniform bivariate distribution of individuals' income and location. With proportional taxation, there is no policy equilibrium with simultaneous voting. We offer a complete characterization of the equations describing the sequential equilibrium in the general case and we show why and how our results depart from those most-preferred by the median income individual located at the median distance from the median. We also compare these majority voting allocations with the socially optimal one.
\end{abstract}

Keywords: proportional income taxation, bidimensional policy and trait spaces. JEL Codes: D72, H41 


\section{Introduction}

Our main objective in this paper is to contribute to the analysis of majority voting over public good provision when both the policy space and the space of voters' traits are multidimensional. Models of democratic public good provision are of interest by themselves, since they shed light on the determinants of the size and type of public goods offered in democracies. Such models are also at the center of the emerging literature on nation formation, whose main objective is to understand the determinants of the number, size and stability of nations. Although our paper does not attempt to introduce such considerations, surveying this literature allows us to take stock of how public good provision under majority voting has been analyzed and to improve upon the models developed.

Contributions to this literature differ according to several dimensions. First, they either deal with the case where the policy choice is horizontal (with citizens selecting the location of their capital or the proportion of a fixed budget to be allocated to a specific public good), vertical (with citizens typically choosing the quantity of a public good), or where both the horizontal and vertical components are voted upon. Second, the citizens may be heterogeneous in their preferences for the public good, in their income, or in both. Papers differ also in whether the distribution of the source of heterogeneity (income and/or preferences) is restricted to be uniform, or whether more general distribution functions are considered. Finally, they differ in how the public good is to be financed: by a lump sum tax or by a proportional income tax. Table 1 summarizes how articles differ according to these dimensions.

[Insert Table 1 around here]

Our paper generalizes the existing literature by incorporating simultaneously the following characteristics: we study the determination by majority voting of both the (horizontal) type and (vertical) size of a public good, when voters differ both in income and in their preferences for the type (i.e., location) of good provided. The distribution of voters' traits is given by a generic bivariate distribution function (i.e., we go beyond the uniform distribution case). We also study both the simultaneous and the sequential determinations of the type and size of the public good.

We now explain why the generalizations we propose are relevant and how the results we obtain qualify and extend those obtained in the literature. Decisions regarding the type and size of the public good to be provided are obviously closely linked and would be better 
understood with a simultaneous voting model. Unfortunately, moving to more than one dimension leads to a discontinuous leap in complexity, as it is well known that simultaneous majority voting on multidimensional policy spaces generically has no equilibrium. ${ }^{1}$ Our first objective is to clarify under which circumstances (i.e., characteristics of the bivariate distribution of individual traits) a majority voting (or Condorcet) equilibrium exists when voting simultaneously over the type and size of the public good. The answer to this question depends on the type of public good financing considered: lump sum or with a proportional income tax. In the first case, we obtain that a majority voting equilibrium exists only if the distribution of preferences in the economy is median uniform. This condition, which we precisely define, is very stringent (although satisfied by the uniform distribution) and non generic. In the case of proportional income taxation, a majority voting equilibrium never exists.

These results explain why the few papers (Alesina et al. (1999), Etro (2006), Gregorini (2009), Perroni and Scharf (2001) $)^{2}$ studying both public good dimensions assume that majority voting is sequential. At first glance, a sequential mechanism looks safe from the point of view of existence as soon as preferences are regular enough (in particular, singlepeaked on each dimension) since each vote is unidimensional. Note however that, unless its is assumed that the two components are totally separable, the backward resolution will lead to a reduced utility function in the first stage which need not be single-peaked. In order to circumvent this difficulty, existing papers make additional assumptions. First, they all consider an ordering of the votes (first on size, then on type) which guarantees the existence of an equilibrium: Alesina et al. (1999) recognize for instance that "this assumption is made for tractability, in order to avoid issues of multidimensional voting, which is not our focus." Second, most papers restrict themselves to lump sum taxation. In our paper with income and preference heterogeneity, lump sum financing results in the median location being chosen together with the size most-preferred by the individual with the median distance to the median agent. This is the same result as the one obtained by Alesina et al. (1999), Perroni and Scharf (2001) and Etro (2006), which all consider that agents do not differ in income. This shows that introducing income heterogeneity has no impact on the results in the presence of lump sum taxation.

We then study the financing of the public good through proportional income taxation. Such an assumption is much closer to practice than lump sum financing. Observe that

\footnotetext{
${ }^{1}$ More precisely, the set of regular (in particular convex) preference profiles with a majority equilibrium in multidimensional policy spaces is generically empty (Banks and Austen-Smith (1999)).

${ }^{2}$ See also Alesina, Baquir and Hoxby (2004).
} 
citizens' income does matter in such a context, as people varying in income favor different quantities of public good even if they have the same preference for its location. Also, as people differ in two dimensions, we describe the polity by a two-dimensional statistical distribution that need not be uniform on any dimension. ${ }^{3}$ Assuming uniformity is indeed a very special case and makes it difficult for the reader to assess which results (such as the features of the equilibrium policy) may be generalized to other distributions. Also, assuming uniformity eliminates the possibility of discussing the impact of some societal characteristics like for instance polarization (Haimanko, Le Breton and Weber (2005)) or correlation between taste and income on the policy outcome.

We show that, while the choice of the type of public good follows the traditional median logic, the majoritarian determination of the taxation rate is more subtle and need not coincide with the preferences of a citizen with a median trait. More precisely, we take as benchmark the natural extension of the sequential equilibrium identified by Alesina et al. (1999) in a lump sum setting (median location together with public good amount favored by the individual with the median distance to the median location and the median income level) and we show that this benchmark corresponds to the sequential equilibrium with proportional taxation if (i) the distributions of income and of location are independent from each other, (ii) the location distribution is uniform, and (iii) the income distribution is symmetrical. In that case, the sequential voting equilibrium and the benchmark both lead to the socially optimal public good's level and location. If only the first two assumptions are satisfied, then the size of the public good is smaller (resp., larger) than the level identified by this benchmark if the income distribution is everywhere concave (resp., convex). For instance, if income follows a Beta distribution that is positively skewed (as in all OECD countries), then the equilibrium public good level is lower than the one identified by our benchmark, while both levels are larger than socially optimal. As for the correlation between income and location preferences, we investigate numerically the case where both distributions are uniform. We obtain that the benchmark corresponds to the case where they are either perfectly correlated or independent from each other. In the case where both traits are positively but imperfectly correlated, the equilibrium public good level is lower than the one identified by this benchmark, which is socially optimal. ${ }^{4}$

\footnotetext{
${ }^{3}$ We follow the same approach as the one adopted by Le Breton and Weber (2003) in the traditional unidimensional model.

${ }^{4}$ It is difficult to compare our results with the two other papers studying proportional taxation. Bolton and Roland (1997) assume that people differ only in income and vote over the size of the public good. They obtain the classical result that the voter with the median income is decisive. Gregorini (2009) introduces heterogeneity in both preferences and income but only considers two income groups. Moreover, he assumes that the public good amount is determined by a social planner rather than by majority voting.
} 
The paper is structured as follows. Section 2 presents the model. Section 3 studies the case where the public good is financed by lump sum taxation while section 4 is devoted to the financing with proportional income taxation. In both sections, we start with the simultaneous voting game before analyzing the sequential voting game. Section 5 compares the equilibrium allocation with sequential voting with the benchmark allocation obtained from Alesina et al. (1999) and with the socially optimal allocation. We also show in this section how equilibrium allocations are impacted by the shape of the income distribution and by its correlation with the location distribution.

We now turn to the setting of our model.

\section{The Model}

We consider an economy populated by a continuum of citizens of unitary mass. This economy has to select a public policy consisting of two components: a horizontal component, which is described as a continuous variable in the interval $[0,1]$, and a vertical component described as a continuous variable in $\Re_{+}$. While the model can accommodate several alternative interpretations, it is useful to think of the public policy as a decision on both the type $p$ of a pure public good, facility or service to produce (the horizontal dimension) and on the quantity or size $g$ of this particular public good (the vertical dimension). A nice illustration is the case where the horizontal dimension is simply the location of the public good.

Citizens are heterogeneous in two dimensions. First, they differ according to their preferences for the different types of public goods. In that respect, each citizen is described by a parameter $\alpha$ in $[0,1]$. Second, each citizen is described by his/her private income $y$ in the interval $[0, \bar{y}] .^{5}$ The statistical distribution of types across citizens is described by a joint distribution $F$ on $[0,1] \times[0, \bar{y}]$ which is assumed to be absolutely continuous with respect to the Lebesgue measure on $\Re^{2}$. We will denote by $f$ its density and by $F_{\alpha}$ and $F_{y}$ the marginal of $F$ on $[0,1]$ and $[0, \bar{y}]$, respectively.

The payoff of an individual of type $(\alpha, y)$ when the policy $(p, g)$ is selected is equal to

$$
V(g)[\lambda-|\alpha-p|]+y-t(y)
$$

where $V^{\prime}>0, V^{\prime \prime}<0, V(0) \geq 0$ and where $\lambda \geq 1$ is a parameter and $t(y)$ denotes the tax paid by such a citizen. Therefore, the parameter $\alpha$ represents the most-preferred type of public good by a citizen with type $\alpha$, irrespective of his/her income. Note also that preferences are

\footnotetext{
${ }^{5}$ This is without loss of generality since we can set $\bar{y}=+\infty$, for instance to consider popular income distributions such as the Pareto and Beta distributions.
} 
quasi-linear with respect to income which is implicitly assumed to be equivalent to private consumption. ${ }^{6}$ We focus on the case where the tax is an affine function of income - i.e.,

$$
t(y)=a y+b,
$$

where $a \in[0,1]$ and $b \in[0, \bar{b}]$. The technology used to produce the public good in quantity $g$ is assumed to exhibit constant returns to scale irrespective of the type of public good which is selected - i.e., up to a normalization, $g$ units of numeraire are needed to produce $g$ units of public good. The government budget constraint is then given by

$$
\begin{aligned}
g & =\int_{0}^{1} d \alpha \int_{0}^{\bar{y}} t(y) f(\alpha, y) d y \\
& =b+a y_{M}
\end{aligned}
$$

where

$$
y_{M} \equiv \int_{0}^{1} d \alpha \int_{0}^{\bar{y}} y f(\alpha, y) d y
$$

is the average income in the economy. ${ }^{7}$

Combining all these elements, we deduce that the (indirect) utility of a citizen of type $(\alpha, y)$ for the policy $(p, a, b)$ is

$$
V\left(b+a y_{M}\right)[\lambda-|\alpha-p|]+(1-a) y-b .
$$

We restrict ourselves to two categories of tax functions belonging to the affine family: pure lump sum taxation $(b>0, a=0)$ and pure proportional taxation $(b=0, a>0) .{ }^{8}$ We start with the simpler case, lump sum taxation, then moving to proportional taxation, where additional effects crop up. In both cases, we first assume that individuals vote simultaneously over the type and the size of the public good. We show that the conditions (on the distribution of traits) to have an equilibrium (a Condorcet winning policy pair -i.e., a

\footnotetext{
${ }^{6}$ Most contributions to the nation formation literature consider a quasi-linear setting. This simplifying assumption has two important implications. First, it simplifies the analysis as we rule out direct income effects (preferences for public goods are unrelated to income levels). Second, it implies that (Pareto) efficiency is equivalent to the maximization of social surplus which is here $V(g)\left[\int_{0}^{1}[\lambda-|\alpha-p|] F_{\alpha} d \alpha\right]-g$. We come back to the optimal allocation in section 5 .

${ }^{7}$ All our results are robust to the introduction of a distortionary cost of taxation as in Bolton and Roland (1997), in the form of a small quadratic cost that decreases each individual's tax proceeds by $\delta \frac{a^{2}}{2} y$ where $\delta$ is a positive parameter.

${ }^{8}$ We leave the analysis of the (simultaneous or sequential) determination of the more general 3-parameter $(a, b$ and $p)$ model for future research.
} 
policy pair that is preferred by a majority of voters to any other feasible policy pair) are very restrictive. We next introduce a sequential way to choose the two policies, assuming that people vote first over the tax policy and then over the type of public good. This sequence of votes seems reasonable and is indeed the one most often studied in the literature. ${ }^{9}$ We identify the equilibrium policy pair under this sequence of votes, and we study how it is affected by the bivariate distribution of voters' traits.

\section{Lump sum taxation}

In this section, we consider the case analyzed by Alesina et al. (1999) and Etro (2006) where $t(y)=b$ - i.e., a tax which is the same for all citizens. The (indirect) utility of a citizen of type $(\alpha, y)$ for the policy $(p, b)$ is ${ }^{10}$

$$
U(p, b)=V(b)[\lambda-|\alpha-p|]+y-b .
$$

We see immediately that the individual's income plays no role in determining his preferences for either $b$ or $p$. Obviously, individuals' most-preferred policy position $p$ corresponds to their own position $\alpha$. As for their most-preferred lump sum tax $b$, conditional on $p=\alpha$, it is given by the following first-order condition

$$
\lambda V^{\prime}(b)=1
$$

and is thus the same for all individuals. Figure 1 illustrates the preference upper contour sets of four individuals, when $V(b)=\sqrt{b}$ and $\lambda=3 / 2$. Comparing the top left panel with the two bottom ones (where individuals differ in income but share the same position $\alpha$ ) confirms that the upper contour sets are not affected by the individual's income level. Comparing the two top panels allows to see the impact of modifying the individual's position $\alpha$ on his preferences.

Insert Figure 1 around here

We first study the simultaneous determination of $p$ and $b$.

\footnotetext{
${ }^{9}$ Alesina et al. (1999) motivate the order of this sequence by arguing that it "resembles common budget procedures in which the size of the budget is decided before its composition." The opposite voting sequence, when type is chosen before size, is more complex, because the public good's type has an impact on individuals' most-preferred size. We compare the two sequential voting procedures with lump sum taxation in De Donder et al. (2011).

${ }^{10} \mathrm{We}$ assume that individuals have enough income or (unmodelled) wealth to pay any lump sum transfer lower than or equal to $\bar{b}$.
} 


\subsection{Simultaneous vote over both policy dimensions}

Our objective in this section is to assess under which circumstances (i.e., distributions of individuals' traits) a Condorcet winning pair $(p, b)$ exists when voting simultaneously over $p$ and $b$. We proceed as follows. In this section, we assume that the indirect utility functions of the citizens are strictly quasi-concave (in Appendix 1, we show that a sufficient condition for quasi concavity is that $V$ is concave enough and/or $\lambda$ is large enough). Under this assumption, an alternative $(p, b)$ is a Condorcet winner if and only if there exists a neighborhood centered on $(p, b)$ which does not contain an alternative defeating $(p, b)$-i.e., $(p, b)$ is a Condorcet winner if and only if $(p, b)$ is a local or differential Condorcet winner (Banks and Austen-Smith (1999)).

We first characterize the set of voters who would favor a policy change in the generic direction $d$ to some given policy bundle. By looking at a specific direction, we show that any equilibrium policy must entail the provision of the public good with the median mostpreferred location. Using this information, we characterize the tax component of the equilibrium policy as well. Finally, by looking at all possible deviations, we show that the conditions that the bivariate distribution of characteristic $F$ must satisfy for a Condorcet winner to exist are very restrictive, and satisfied mainly by the uniform distribution.

Starting from a generic policy bundle $(p, b)$, the individuals who (weakly) favor a deviation in the direction $d$ are such that

$$
\left\langle d,\left(\frac{\partial U(p, b)}{\partial p}, \frac{\partial U(p, b)}{\partial b}\right)\right\rangle \geq 0,
$$

where $\langle.,$.$\rangle denotes the scalar product and where { }^{11}$

$$
\begin{aligned}
\frac{\partial U(p, b)}{\partial b} & =V^{\prime}(b)[\lambda-|\alpha-p|]-1, \\
\frac{\partial U(p, b)}{\partial p} & =V(b) \text { if } \alpha>p \\
& =-V(b) \text { if } \alpha<p, \\
& =0 \text { if } \alpha=p .
\end{aligned}
$$

If we denote by $d_{p}$ the horizontal component of the vector $d$, and by $d_{b}$ its vertical component, we obtain that the set of individuals favoring the direction $d$ (starting from

\footnotetext{
${ }^{11}$ Strictly speaking, the function $U$ is not differentiable with respect to $p$ when $p=\alpha$, but since $p=\alpha$ corresponds to the peak of the function, we set its derivative equal to zero. Not having to deal explicitly with the non-differentiability at one point allows us to simplify a lot the exposition of the results, without of course affecting them.
} 
$(p, b))$ is given by $^{12}$

$$
\begin{aligned}
& \left\{(\alpha, y) \text { such that } \alpha>p \text { and } d_{p} V(b)+d_{b}\left[V^{\prime}(b)[\lambda-\alpha+p]-1\right] \geq 0\right\} \\
& \cup\left\{(\alpha, y) \text { such that } \alpha<p \text { and }-d_{p} V(b)+d_{b}\left[V^{\prime}(b)[\lambda-p+\alpha]-1\right] \geq 0\right\} .
\end{aligned}
$$

If we take a direction such that $d_{p}>0$ and $d_{b}=0$ (i.e., an increase in $p$ without modification of $b$ ), we obtain the straightforward result that all individuals with $\alpha<p$ oppose this move while all those with $\alpha>p$ favor this move. We then obtain that, to be immune to deviations in this direction, the starting pair must be such that $p=\alpha_{m e d}$, with $\alpha_{\text {med }}$ the unique solution of the equation

$$
F_{\alpha}(x)=\frac{1}{2}
$$

In words, any public good location different from the median most-preferred one in the population would be defeated by a proposal moving this location closer to the median.

From now on, we assume that $p=\alpha_{\text {med }}$ as initial location and we focus on the tax component of the policy bundle. If we consider a deviation $d$ such that $d_{b}>0$, we obtain that the set of individuals (weakly) favoring this deviation from $\left(\alpha_{m e d}, b\right)$ is given by

$$
\begin{aligned}
& \left\{(\alpha, y) \text { such that } \alpha>\alpha_{m e d} \text { and } \alpha \leq \alpha_{m e d}+\lambda+\frac{d_{p} V(b)}{d_{b} V^{\prime}(b)}-\frac{1}{V^{\prime}(b)}\right\} \\
& \cup\left\{(\alpha, y) \text { such that } \alpha<\alpha_{m e d} \text { and } \alpha \geq \alpha_{m e d}-\lambda+\frac{d_{p} V(b)}{d_{b} V^{\prime}(b)}+\frac{1}{V^{\prime}(b)}\right\} .
\end{aligned}
$$

It is convenient to denote by $\beta$ the variable $\lambda-\left|\alpha-\alpha_{\text {med }}\right|$. Loosely speaking, this variable measures how much an individual located at $\alpha$ values a public good of type $\alpha_{\text {med }}$. Note that $\beta$ varies in the interval $[\eta, \lambda]$ where $\eta \equiv \lambda-\operatorname{Max}\left(\alpha_{\text {med }}, 1-\alpha_{\text {med }}\right)$. Formally, let $H$ denote the joint distribution of $(\beta, y)$ in $[\eta, \lambda] \times[0, \bar{y}]$ and let $H_{\beta}$ and $H_{y}$ be the corresponding two marginal distributions. We have

$$
H_{\beta}(x)=F_{\alpha}\left(x-\lambda+\alpha_{m e d}\right)+\left[1-F_{\alpha}\left(\lambda+\alpha_{m e d}-x\right)\right]
$$

leading to the first marginal density

$$
h_{\beta}(x)=f_{\alpha}\left(x-\lambda+\alpha_{m e d}\right)+f_{\alpha}\left(\lambda+\alpha_{m e d}-x\right) .
$$

\footnotetext{
${ }^{12}$ The assumption that $F$ is absolutely continuous with respect to the Lebesgue measure guarantees that the set of individuals with $\alpha=\alpha_{\text {med }}$ has zero measure. To simplify notation and save space, we restrict ourselves to the description of sets with strictly positive measure.
} 
We can now express (4) in a more concise way:

$$
\begin{aligned}
& \left\{(\alpha, y) \text { such that } \alpha>\alpha_{\text {med }} \text { and } \beta \geq \frac{1}{V^{\prime}(b)}-\frac{d_{p} V(b)}{d_{b} V^{\prime}(b)}\right\} \\
& \cup\left\{(\alpha, y) \text { such that } \alpha<\alpha_{m e d} \text { and } \beta \geq \frac{1}{V^{\prime}(b)}+\frac{d_{p} V(b)}{d_{b} V^{\prime}(b)}\right\} .
\end{aligned}
$$

If $d_{p}=0$ and $d_{b}>0$, we obtain that the individuals who favor the direction $d$ are characterized by

$$
\beta \geq \frac{1}{V^{\prime}(b)} .
$$

It is easy to interpret this inequality. Observe from (3) that the utility of individual $(\beta, y)$ when $p=\alpha_{m e d}$ is

$$
U\left(\alpha_{m e d}, b\right)=\beta V(b)+y-b .
$$

The first-order condition for $b$ is then given by

$$
\beta V^{\prime}(b)-1=0 \text {. }
$$

Condition (5) then means that all individuals whose most-preferred lump sum tax is larger than $b$ support a move in any direction $d$ that increases $b$ with $p$ constant $\left(d_{p}=0, d_{b}>0\right)$. By contrast, individuals whose most-preferred lump sum tax is strictly lower than $b$ would support a move in the opposite direction, $-d$. For a majority of voters to prefer $b$ to a move in either direction $d$ or $-d$, we must have

$$
\begin{aligned}
\beta_{\text {med }} V^{\prime}(b) & =1 \\
\Leftrightarrow b & =V^{\prime^{-1}}\left(\frac{1}{\beta_{\text {med }}}\right),
\end{aligned}
$$

where $\beta_{\text {med }}$ is the median value of $\beta$ :

$$
H_{\beta}\left(\beta_{\text {med }}\right)=\frac{1}{2}
$$

We have thus proved the following:

Proposition 1 Assume that $t(y)=b$ and that individuals vote simultaneously over $p$ and $b$. Then, if a Condorcet winning pair $(p, b)$ exists, $p=\alpha_{\text {med }}$ and $b$ is the most-preferred lump sum tax of the individuals with the median distance to the median $\alpha$.

It is interesting to note that the most-preferred lump sum tax of the median individual (such that $\alpha=\alpha_{m e d}$, or $\beta=\lambda$ ) is not part of the Condorcet winning policy pair. Indeed, 
it is easy to see that voters (other than $\alpha_{m e d}$ ) unanimously support a decrease in taxation from the policy $\left(\alpha_{m e d}, V^{\prime-1}\left(\frac{1}{\lambda}\right)\right)$. The reason for this is intuitive: since $p=\alpha_{m e d}$, the median individual $\alpha_{\text {med }}$ obtains exactly the kind of public good he most prefers. He is then the individual whose marginal valuation of the public good is the largest in the polity. In other words, if this voter has his say on the lump sum tax, everyone would like to decrease this tax because they all value the public good less (at the margin) than him.

We now turn to the conditions under which the policy pair $\left(\alpha_{m e d}, V^{\prime^{-1}}\left(\frac{1}{\beta_{m e d}}\right)\right)$ is a Condorcet winner. A first necessary condition is that, whatever $d_{p}$ and for any $d_{b}>0$, the set of people who (weakly) favor a move in the direction $d$ has a measure at most equal to one half. This set of individuals is given by (4) where we replace $b$ by $V^{\prime^{-1}}\left(\frac{1}{\beta_{\text {med }}}\right)$ to obtain

$$
\begin{aligned}
& \left\{(\alpha, y) \text { such that } \alpha>\alpha_{\text {med }} \text { and } \alpha \leq \phi_{1}\left(d_{p}, d_{b}\right)\right\} \\
& \cup\left\{(\alpha, y) \text { such that } \alpha<\alpha_{\text {med }} \text { and } \alpha \geq \phi_{2}\left(d_{p}, d_{b}\right)\right\},
\end{aligned}
$$

where

$$
\begin{aligned}
& \phi_{1}\left(d_{p}, d_{b}\right)=\operatorname{Max}\left(\alpha_{\text {med }}+\left(\lambda-\beta_{\text {med }}\right)+\beta_{\text {med }} \frac{d_{p}}{d_{b}} V\left(V^{\prime^{-1}}\left(\frac{1}{\beta_{\text {med }}}\right)\right), \alpha_{\text {med }}\right), \\
& \phi_{2}\left(d_{p}, d_{b}\right)=\operatorname{Min}\left(\alpha_{\text {med }}-\left(\lambda-\beta_{\text {med }}\right)+\beta_{\text {med }} \frac{d_{p}}{d_{b}} V\left(V^{\prime^{-1}}\left(\frac{1}{\beta_{\text {med }}}\right)\right), \alpha_{\text {med }}\right) .
\end{aligned}
$$

The first necessary condition is then that the measure of this set equals at most one half:

$$
F_{\alpha}\left(\phi_{1}\left(d_{p}, d_{b}\right)\right)-F_{\alpha}\left(\phi_{2}\left(d_{p}, d_{b}\right)\right) \leq \frac{1}{2}
$$

To shed some light on this condition, we first assume that $d_{p}=0$. In that case, condition (7) simplifies to

$$
F_{\alpha}\left(\phi_{1}\left(0, d_{b}\right)\right)-F_{\alpha}\left(\phi_{2}\left(0, d_{b}\right)\right) \leq \frac{1}{2}
$$

where

$$
\begin{aligned}
& \phi_{1}\left(0, d_{b}\right)=\alpha_{m e d}+\left(\lambda-\beta_{m e d}\right)>\alpha_{m e d} \\
& \phi_{2}\left(0, d_{b}\right)=\alpha_{m e d}-\left(\lambda-\beta_{m e d}\right)<\alpha_{m e d}
\end{aligned}
$$

In that case, the set of voters who prefer the direction $d$ (i.e., an increase in $b$ without change in the location) is given by an interval of people centered on the individual with the median most-preferred location. Recall that people close to the median location have the highest willingness to pay for this kind of good - it is thus no surprise that an interval of people around that median prefer a higher value of $b$. The fact that this interval is exactly 
centered on $\alpha_{\text {med }}$ crucially depends on the assumption that $d_{p}=0$ : in that case, people equidistant from $\alpha_{m e d}$ have exactly the same preference for an increase in $b$.

If we rather consider that $d_{p}>0$ (together with $d_{b}>0$ ), the interval of people who prefer an increase in $b$ moves to the right: distance from $\alpha_{m e d}$ is not the only thing that matters anymore, since people to the right of $\alpha_{\text {med }}$ benefit from $d_{p}>0$ while people to the left of $\alpha_{m e d}$ dislike this component of $d$. As $d_{p}$ becomes large compared to $d_{b}, \phi_{2}\left(d_{p}, d_{b}\right)$ becomes larger than $\alpha_{m e d}$ and the only people favoring such a move have a larger-than-average value of $\alpha$. Similarly, if we consider $d_{p}<0$ (together with $d_{b}>0$ ), the interval of people who favor direction $d$ shifts to the left, and as $d_{p} / d_{b}$ becomes sufficiently large (in absolute value), $\phi_{1}\left(d_{p}, d_{b}\right)$ becomes smaller than $\alpha_{m e d}$ and only people with lower-than-average values of $\alpha$ are in favor of direction $d$.

When $d_{b}<0$, the set of people who (weakly) favor a move in the direction $d$ is given by

$$
\begin{aligned}
& \left\{(\alpha, y) \text { such that } \alpha>\alpha_{\text {med }} \text { and } \alpha \geq \phi_{1}\left(d_{p}, d_{b}\right)\right\} \\
& \cup\left\{(\alpha, y) \text { such that } \alpha<\alpha_{\text {med }} \text { and } \alpha \leq \phi_{2}\left(d_{p}, d_{b}\right)\right\},
\end{aligned}
$$

which is the complement to set (6). The necessary and sufficient condition for $(p, b)$ to be a Condorcet winning pair is then that both sets (6) and (8) have a measure at most equal to one half, with translates to

$$
F_{\alpha}\left(\phi_{1}\left(d_{p}, d_{b}\right)\right)-F_{\alpha}\left(\phi_{2}\left(d_{p}, d_{b}\right)\right)=\frac{1}{2} .
$$

By definition of $\beta_{\text {med }}$, condition (9) is satisfied when $d_{p}=0$. On the other hand, this measure will generically move away from one half as $d_{p}$ is increased (for any constant $d_{b}$ ), except in very special circumstances. Note that condition (9) can be reformulated as

$$
\left.\left.F_{\alpha}\left(\alpha_{m e d}+\widehat{d}+d\right)-F_{\alpha}\left(\alpha_{m e d}-\widehat{d}+d\right)=\frac{1}{2} \text { for all } d \in\right]-\widehat{d}, \widehat{d}\right],
$$

where $F_{\alpha}\left(\alpha_{m e d}\right)=\frac{1}{2}$ and $\widehat{d}$ is such that $F_{\alpha}\left(\alpha_{\text {med }}+\widehat{d}\right)-F\left(\alpha_{\text {med }}-\widehat{d}\right)=\frac{1}{2}$. When $d=\widehat{d}$, this implies $F_{\alpha}\left(\alpha_{\text {med }}+2 \widehat{d}\right)-F_{\alpha}\left(\alpha_{\text {med }}\right)=\frac{1}{2}$ and therefore $F_{\alpha}\left(\alpha_{\text {med }}+2 \widehat{d}\right)=1$. Similarly, when $d=-\widehat{d}$, this implies $\left.F_{\alpha}\left(\alpha_{\text {med }}\right)-F_{\alpha}\left(\alpha_{\text {med }}-2 \widehat{d}\right)\right)=\frac{1}{2}$ and therefore $F_{\alpha}\left(\alpha_{\text {med }}+2 \widehat{d}\right)=0$. Hereafter, the distributions satisfying (10) are called median uniform. We have then proved:

Proposition 2 Assume that $t(y)=b$ and that individuals vote simultaneously over $p$ and b. A Condorcet winning pair $(p, b)$ exists only if the distribution of $\alpha$ is median uniform.

The uniform distribution is of course median uniform, but there are other examples. Take for instance any function $h$ on $\left[\frac{1}{4}, \frac{3}{4}\right]$ such that $h(t)=h(1-t)$ for all $t \in\left[\frac{1}{4}, \frac{1}{2}\right]$ and 
$\int_{\frac{1}{4}}^{\frac{1}{2}} h(t) d t=\frac{1}{4}$. Let $f$ be the function defined on $[0,1]$ as

$$
f(\alpha)=\left\{\begin{array}{c}
h\left(\frac{1}{2}-t\right) \text { for all } t \in\left[0, \frac{1}{4}\right] \\
h(t) \text { for all } t \in\left[\frac{1}{4}, \frac{3}{4}\right] \\
h\left(\frac{3}{2}-t\right) \text { for all } t \in\left[\frac{3}{4}, 1\right]
\end{array}\right.
$$

It is straightforward to check that $f$ is the density of a median uniform distribution.

Despite some flexibility, it should however be clear that median uniformity is not generic i.e., the condition is violated for some small perturbations of any median uniform distribution. Since a Condorcet winning pair $(p, b)$ generically does not exist, we consider in the next section a sequential determination of the two policy dimensions.

\subsection{Sequential vote}

In this section, we proceed as in Alesina et al. (1999) and consider a sequential majority procedure where citizens vote first on the lump sum $\operatorname{tax} b$ and then on the type $p$. It is immediate to see from (3) that there is a majority equilibrium in the second stage which is independent of the decision on $b$ in the first stage. This majority equilibrium corresponds to the median value $\alpha_{\text {med }}$ of $\alpha$.

Solving backward, in the first stage the individuals anticipate the value of $p$ that will be chosen in the second stage and vote according to their utility function (3) where we make use of $p=\alpha_{m e d}$ and of the definition of $\beta$ to obtain

$$
U\left(\alpha_{m e d}, b\right)=\beta V(b)+y-b .
$$

This utility function is concave in $b$, and individual $\beta$ 's most-preferred value of $b$ (given that $\left.\alpha=\alpha_{\text {med }}\right)$, which we denote by $b^{*}(\beta)$, is the solution to

$$
\beta V^{\prime}(b)-1=0
$$

in the case of an interior solution. We have that

$$
\begin{aligned}
& b^{*}(\beta)=0 \text { if } \beta V^{\prime}(0)<1-\text { i.e., if } \beta \text { is low enough and } \\
& b^{*}(\beta)=\bar{b} \text { if } \beta V^{\prime}(\bar{b})>1-\text { i.e., if } \beta \text { is large enough. }
\end{aligned}
$$

Using the implicit function theorem, we obtain that

$$
\frac{\partial b^{*}(\beta)}{\partial \beta}=-\frac{V^{\prime}(b)}{\beta V^{\prime \prime}(b)}>0 .
$$


The sensitivity of the most-preferred tax of an individual to his distance to the median type depends on the shape of $V$.

Figure 2 illustrates the iso-tax lines in the $(\beta, y)$ space. They are vertical, since the income dimension does not play any role here.

Insert Figure 2 around here

Applying the usual median voter theorem, we obtain that the result of the vote is $b=$ $b^{*}\left(\beta_{\text {med }}\right)$ - i.e., the lump sum tax most-preferred by the individuals located at the median distance from the median, which is the rule derived by Alesina et al. (1999). Note that, in stark contrast with the preceding section, a sequential equilibrium always exists, whatever the distribution $F$.

We have obtained the following proposition.

Proposition 3 Assume that $t(y)=b$ and that individuals vote sequentially, first over the lump sum tax $b$ and then over the public good type $p$. The unique equilibrium of this voting procedure is given by $p=\alpha_{\text {med }}$ and $b=b^{*}\left(\beta_{\text {med }}\right)$ - i.e., the chosen type is the median one, while the lump sum tax is the one most-favored by the individuals with the median distance from the median on the location dimension.

Corollary 1 The Condorcet winning pair $(p, b)$ obtained with simultaneous voting over $p$ and $b$, if it exists, is identical to the equilibrium of the sequential voting game where $b$ is chosen first and $p$ second.

When the public good is financed with a lump sum transfer, the income heterogeneity among individuals plays absolutely no role. We now turn to the richer case where proportional income taxes are used.

\section{Proportional income taxation}

In the case of a proportional income tax $t(y)=a y$, the (indirect) utility of a citizen of type $(\alpha, y)$ for the policy $(p, a)$ is

$$
W(p, a)=V(g(a))[\lambda-|\alpha-p|]+(1-a) y
$$


where $g(a)$ is given by the government budget constraint (2) when $b=0$. We proceed as in the previous section: we first show that there is in general no Condorcet winner when individuals vote simultaneously over $p$ and $a$. We then study the sequential game where individuals vote first over $a$ and then over $p$. We provide in Appendix 2 the proof that indirect utilities are quasi-concave with proportional income taxation if $V$ is concave enough and/or $\lambda$ is large enough. We then assume quasi-concavity of indirect utility preferences in this section.

\subsection{Simultaneous vote over $p$ and $a$}

We start by looking at the optimal policy $\left(p^{*}(\alpha, y), a^{*}(\alpha, y)\right)$ of a citizen of type $(\alpha, y)$. Clearly, $p^{*}(\alpha, y)=\alpha$ : whatever the size of the public good, the individual would like its favored type to be provided. We then obtain that $a^{*}(\alpha, y)$ is solution to the following firstorder condition

$$
V^{\prime}(g(a))=\frac{y}{\lambda y_{M}} .
$$

It is immediate to see from (14) that the most-preferred tax rate of an individual now depends on his income, in sharp contrast with the lump sum financing case. Figure 3 depicts the preference upper contour sets of four individuals when $V(b)=\sqrt{b}$ and $\lambda=3 / 2$. Not surprisingly, the most-preferred tax rate is decreasing with the individual's income, since taxes owed increase with income.

\section{Insert Figure 3 around here}

We proceed as in the previous section, starting from a generic policy bundle $(p, a)$ and looking for necessary conditions that its components have to satisfy in order for the pair to be a Condorcet winner. Starting from $(p, a)$, the individuals who favor a deviation in the direction $d$ are such that

$$
\left\langle d,\left(\frac{\partial W(p, a)}{\partial p}, \frac{\partial W(p, a)}{\partial a}\right)\right\rangle \geq 0
$$

where

$$
\begin{aligned}
\frac{\partial W(p, a)}{\partial a} & =V^{\prime}(g(a)) y_{M}[\lambda-|\alpha-p|]-y, \\
\frac{\partial W(p, a)}{\partial p} & =V(g(a)) \text { if } \alpha>p \\
& =-V(g(a)) \text { if } \alpha<p \\
& =0 \text { if } \alpha=p .
\end{aligned}
$$


We denote as previously by $d_{p}$ the horizontal component of the vector $d$, and by $d_{a}$ its vertical component. The set of individuals favoring the direction $d$ (starting from $(p, a)$ ) is given by

$$
\begin{aligned}
& \left\{(\alpha, y) \text { such that } \alpha>p \text { and } d_{p} V(b)+d_{a}\left[V^{\prime}(g(a)) y_{M}[\lambda-\alpha+p]-y\right] \geq 0\right\} \\
& \cup\left\{(\alpha, y) \text { such that } \alpha<p \text { and }-d_{p} V(b)+d_{a}\left[V^{\prime}(g(a)) y_{M}[\lambda-p+\alpha]-y\right] \geq 0\right\} .
\end{aligned}
$$

We use the same argument as in the preceding section to show that a Condorcet winning policy pair must involve $p=\alpha_{\text {med }}$ : if it were not the case, a majority of voters would like to deviate in the direction of the median $\alpha$ while keeping the tax rate $a$ constant.

From now on, we assume that $p=\alpha_{\text {med }}$ and focus on the tax component of the policy bundle. If we consider a deviation $d$ such that $d_{a}>0$, we obtain that the set of individuals favoring this deviation from $\left(\alpha_{m e d}, a\right)$ is given by

$$
\begin{aligned}
& \left\{(\alpha, y) \text { such that } \alpha>\alpha_{m e d} \text { and } y \leq \beta V^{\prime}(g(a)) y_{M}+\frac{d_{p}}{d_{a}} V(g(a))\right\} \\
& \cup\left\{(\alpha, y) \text { such that } \alpha<\alpha_{m e d} \text { and } y \leq \beta V^{\prime}(g(a)) y_{M}-\frac{d_{p}}{d_{a}} V(g(a))\right\} .
\end{aligned}
$$

If $d_{p}=0$, we obtain that all individuals who (weakly) favor the direction $d$ (an increase in $b$ while keeping $p$ constant) are such that

$$
y \leq \beta V^{\prime}(g(a)) y_{M}
$$

Observe that the first-order condition for an individual $(\beta, y)$ faced with $p=\alpha_{m e d}$ is given by

$$
y=\beta V^{\prime}(g(a)) y_{M} .
$$

To illustrate the joint effect of $y$ and $\beta$ on the optimal choice, we denote by $\tilde{y}(\beta, a)$ the income level that satisfies (17) -i.e., the income of an individual of type $\beta$ who most prefers a proportional tax rate of $a$ when faced with $p=\alpha_{\text {med }}$. Condition (16) shows that all individuals who have an income lower than $\tilde{y}$ are in favor of an increase in $a$ (i.e., $d_{a}>0$ ). Figure 4 (a) illustrates condition (16) in the $(\alpha, y)$ space. Assume that we start with the value of $a$ that is most-preferred by the individual with the median income and the median location, so that $\tilde{y}(\lambda, a)=y_{\text {med }}$. Among individuals located at $\alpha_{m e d}$, those who favor an increase in $a$ are those whose income is lower than the median income in the population. Individuals located further from $\alpha_{\text {med }}$ on the horizontal axis have a lower marginal valuation of the public good. As the benefit from this good decreases with the distance between individual location $\alpha$ and median location, so does the threshold value of income below 
which voters favor an increase in the tax rate. To satisfy the optimal behavior described by (17), $\beta$ and $y$ must be comonotonic (in other words, they must change in the same direction).

Insert Figure 4 around here

One immediately infers from this figure that the policy bundle composed of $\alpha_{\text {med }}$ and of the most-preferred tax rate of the individual with both the median location and the median income is always defeated by a majority of voters who prefer a lower tax rate (and the same location). The intuition is similar to the one exposed in the lump sum case, and is based upon the observation that individuals with the median location are those who have the largest direct benefits from the public good. Consequently, a move to decrease the tax rate from their most-preferred level is favored not only by all individuals with above-median income, but also by poorer-than-median individuals who are located far enough from the median location. This holds true whatever the distribution function $F$ or $H$.

Let us denote by $\left(\alpha_{m e d}, a^{\circ}\right)$ the policy pair that is such that exactly one half of the polity is in favor of an increase in the tax rate when the location of the public good is kept unchanged. It is clear from above that this policy is the most-preferred one of an individual with median location and with a larger-than-median income: $\tilde{y}\left(\lambda, a^{\circ}\right)>y_{m e d}$. For instance, in the case where the distributions of locations and of income are both uniform (and independent), the tax rate $a^{\circ}$ is given by

$$
\int_{\eta}^{\lambda} \beta V^{\prime}\left(g\left(a^{\circ}\right)\right) \frac{y_{M}}{\lambda-\eta} d \beta=1 / 2 .
$$

Making use of the first-order condition of the individual $\tilde{y}\left(\lambda, a^{\circ}\right)$, we obtain that

$$
\tilde{y}\left(\lambda, a^{\circ}\right)=\frac{\lambda}{\lambda+\eta}=\frac{\lambda}{2 \lambda-\frac{1}{2}}
$$

i.e. that $\tilde{y}\left(\lambda, a^{\circ}\right)>y_{m e d}$ as explained above.

It is interesting to note that, in the case of the uniform and independent distributions of $\beta$ and $y$, the tax rate $a^{\circ}$ is also the most-preferred tax of an individual with the average income $y_{M}$ and with the median distance to the median location, $\beta_{\text {med }}: \tilde{y}\left(\beta_{\text {med }}, a^{\circ}\right)=y_{M}$. To prove this, it is enough to observe that the first-order condition for an individual with $\beta=\lambda$ and $y=\tilde{y}\left(\lambda, a^{\circ}\right)$,

$$
\lambda V^{\prime}(g(a)) y_{M}=\frac{\lambda}{2 \lambda-\frac{1}{2}}
$$


is the same as the first-order condition for individual with $\beta=\beta_{\text {med }}$ and $y=y_{M}$

$$
\left(\lambda-\frac{1}{4}\right) V^{\prime}(g(a)) y_{M}=y_{M}
$$

We summarize our results so far in the next Proposition.

Proposition 4 Assume that $t(y)=$ ay and that individuals vote simultaneously over $p$ and a. Then, if a Condorcet winning pair $(p, a)$ exists, it is such that $p=\alpha_{\text {med }}$ and that a is the most-preferred proportional tax of individuals with median location and with a larger-thanmedian income. In the special case of uniform and independent distributions of $\alpha$ and $y$, this policy a is also the one most-preferred by an individual with the average income $y_{M}$ and the median distance to the median location, $\beta_{\text {med }}$.

We now look at the set of people who prefer an increase in taxation coupled with an increase in the location of the public good (i.e., a move $d_{p}>0$ and $d_{a}>0$ ), starting from $\left(\alpha_{m e d}, a\right)$. This set is defined in (15) and is represented on Figure $4(\mathrm{~b})$. The threshold income below which individuals favor a move in the direction $d$ remains decreasing in the distance between individual's location and policy location, as in Figure 4 (a), but we now observe a discontinuity for $\alpha=\alpha_{m e d}=p$. To the left of $\alpha_{m e d}$, individuals do not favor an increase in $p$ as it moves the location of the public good further away from their bliss point, while to the right of $\alpha_{m e d}$ individuals do favor such a move. The size of the discontinuity increases with $d_{p}$, the horizontal component of the move considered (for a given $d_{a}$ ). More precisely, as $d_{p}$ increases the threshold income level decreases by $V(g(a)) / d_{a}$ to the left of $\alpha_{m e d}$ and increases by the same amount $V(g(a)) / d_{a}$ to the right of $\alpha_{m e d}$.

The conclusion we draw from Figure 4 is that the existence of a Condorcet winning policy pair when voting simultaneously over the two dimensions is extremely unlikely. Even if we start from a policy pair $\left(\alpha_{m e d}, a^{\circ}\right)$ such that exactly one half of the polity would like to increase the tax rate while keeping the location constant, when we consider directions where both dimensions are modified simultaneously, Figure 4 (b) shows that the bivariate distribution of $\alpha$ and $y$ must exhibit a lot of symmetry for the policy pair to remain immune to these deviations. Moreover, even in the case of the uniform independent distributions, there is no Condorcet winner. This can be seen from the fact that the income distribution is bounded above and that $\tilde{y}\left(\lambda, a^{\circ}\right)>y_{M}$. This last observation means that, if $d_{p}$ is large enough, then the threshold income of an individual who is located immediately to the right of $\alpha_{m e d}$ reaches the upper bound of the distribution. As is shown on Figure 4 (c), we obtain in that case that a majority of voters favor a decrease in $p$ coupled with a decrease in the tax rate. We then obtain 
Proposition 5 Assume that $t(y)=$ ay and that individuals vote simultaneously over $p$ and $a$. There is no Condorcet winning pair $(p, a)$, even in the case of uniform and independent distributions of $\alpha$ and $y$.

We now turn to the sequential determination of both policy dimensions.

\subsection{Sequential vote}

In what follows, we consider a sequential majority procedure where citizens vote first on the tax rate $a$ and then on the type $p$. We see immediately from (13) that there is a majority equilibrium in the second stage which is independent of the decision on $a$ in the first stage. This majority equilibrium, which we denote by $p_{2}$, corresponds to the median value of $\alpha$ :

$$
p_{2}=\alpha_{m e d}
$$

Solving backward, citizens are aware that their choice of $a$ has no influence at all on the result of the second stage vote. Their most-preferred value of $a$, denoted by $\tilde{a}(\beta, y)$, is then obtained as a solution to the following first-order condition

$$
V^{\prime}(g(a))=\frac{y}{\beta y_{M}}
$$

This first-order condition is sufficient since the second derivative of the indirect utility function

$$
V^{\prime \prime}(g(a))-\delta V^{\prime}(g(a))
$$

is negative. If

$$
\beta V^{\prime}(0) y_{M}-y \leq 0, \text { then } \tilde{a}(\beta, y)=0
$$

and if

$$
\beta V^{\prime}\left(y_{M}\right) y_{M}-y \geq 0, \text { then } \tilde{a}(\beta, y)=1 \text {. }
$$

From the implicit function theorem and the second order conditions, we deduce immediately that, if $\tilde{a}(\beta, y)$ is an interior solution, then

$$
\frac{\partial \tilde{a}(\beta, y)}{\partial \beta}=-\frac{V^{\prime}(g(a)) y_{M}}{\beta V^{\prime \prime}(g(a))}>0,
$$

and

$$
\frac{\partial \tilde{a}(\beta, y)}{\partial y}=\frac{1}{\beta V^{\prime \prime}(g(a))}<0 .
$$

Not surprisingly, citizens endowed with a large income or distant from the median type of public good have a low most-preferred proportional tax rate. From (19) we observe that the 
sensitivity of the most-preferred tax rate to the distance from the median type depends on the curvature of $V$. This also guarantees a further degree of freedom for the model. ${ }^{13}$

Figure 5 depicts the iso-tax curves in the type space $[\eta, \lambda] \times[0, \bar{y}]-$ i.e., the locus of types $(\beta, y)$ whose most-preferred tax policy is $a$.

Insert Figure 5 around here

From (19) and (20), we deduce that the slope $d y / d \beta$ of an iso-tax curve corresponding to $a=\tilde{a}(\beta, y)$ is equal to

$$
V^{\prime}(g(a)) y_{M}
$$

which does not depend on $\beta$ nor on $y$-i.e., the iso-tax curves are affine functions. In order to find the equilibrium tax rate emerging from the first stage vote, we have to find the median iso-tax line - i.e. the iso-tax line that separates the set of types into two halves, with one half located above the line (and favoring a smaller tax rate) and the other half located below (and supporting a larger tax rate). ${ }^{14}$ Formally, the equilibrium first stage tax rate, which we denote by $a_{1}$, is the solution to

$$
\Psi(a)=\int_{\eta}^{\lambda} d \beta \int_{0}^{\beta \varphi(a)} h(\beta, y) d y=\frac{1}{2},
$$

where

$$
\varphi(a) \equiv y_{M} V^{\prime}(g(a))
$$

Proposition 6 Assume that $t(y)=$ ay and that individuals vote first over a and then over p. The equilibrium policy pair is composed of the median policy location $\alpha_{\text {med }}$ together with the proportional rate $a_{1}$ as defined in equation (21).

In the next section, we compare the public good levels and location emerging from this sequential procedure under the proportional and lump sum financing schemes, as well as their socially optimum levels. We also assess the role played by the bivariate distribution of voters' types in these comparisons.

\footnotetext{
${ }^{13}$ In the sense that even if we assume a high $\beta$, meaning that people have similar preferences for location, we can mitigate this effect by selecting an appropriate utility function. See also (12).

${ }^{14}$ Given the absolute continuity of $F$ with respect to the Lebesgue measure, the set of individuals located exactly on the median iso-line has zero measure.
} 


\section{Comparison between equilibrium and optimal allo- cations}

Up to now, we have been mainly concerned with the existence and the characterization of equilibrium public good's type and level under various arrangements. In this section, we concentrate on sequential voting procedures, and we compare the equilibrium public good's type and level obtained with lump sum versus proportional financing to each other and to a normative benchmark. This benchmark is easy to ascertain with quasi-linear preferences: it is straightforward to check that the optimal policy $\left(p^{*}, g^{*}\right)$ is such that $p^{*}=\alpha_{m e d}$ and $g^{*}$ is solution of the equation:

$$
V^{\prime}\left(g^{*}\right)=\frac{1}{\beta_{M}},
$$

where $\beta_{M} \equiv \int_{0}^{1}\left[\lambda-\left|\alpha-\alpha_{\text {med }}\right|\right] F_{\alpha} d \alpha$.

We denote by $g^{L}$ and $g^{P}$ the equilibrium public good levels generated by sequential voting (first over $g$ and then over $p$ ) under lump sum and proportional financing, respectively. We summarize the results obtained up to this point in the first three lines of Table 2. Observe that both sequential procedures defined above result in the socially optimal public good's type, $\alpha_{\text {med }} \cdot{ }^{15}$ The remainder of the section then focuses on a comparison of public good levels, with the objective to shed light on the relationships between $g^{L}, g^{P}$ and $g^{*}$, and on the role played by the distribution of types in this comparison.

[Insert Table 2 around here]

We obtain from Table 2 that the ranking of $g^{L}$ (obtained from equation (11) with $\beta=$ $\beta_{\text {med }}$ ) and $g^{*}$ (obtained from (22)) simply relies on the skewness of the distribution of tastes (i.e., on the comparison of $\beta_{\text {med }}$ with $\beta_{M}$ ). The comparison with $g^{P}$ is more complex because, unlike $g^{L}$ and $g^{*}, g^{P}$ is affected by the income distribution as well as by the taste distribution. As we report in the third line of Table 2, the value of $g^{P}$ is determined by the proportional tax rate $a_{1}$ implicitly defined by equation (21). This tax rate $a_{1}$ is most-preferred by individuals with median preference $\beta_{\text {med }}$ and some income level denoted by $\hat{y}$ and implicitly defined by (18).

In order to compare $g^{P}$ with $g^{L}$ and $g^{*}$, we define in the last row of Table 2 a natural benchmark. This benchmark for the proportional tax rate, which we denote by $a_{m e d}$, corresponds to the most-preferred tax rate of citizens with a median income $y_{m e d}$ and a median

\footnotetext{
${ }^{15}$ This is due to the linear specification of the disutility from consuming a public good's type distant from one's own most-favored type. See also Gregorini (2009) for a normative analysis when taxes are linear.
} 
distance to the median, $\beta_{\text {med }}$ (see equation (18)). The corresponding public good level is then $g\left(a_{m e d}\right)=a_{m e d} y_{M}$. Observe from the above formulas that $g^{P}$ and $g\left(a_{m e d}\right)$ differ only according to the income level of the decisive individual. Comparing the income levels $\hat{y}$ and $y_{m e d}$ under various assumptions regarding the distributions of income and taste will then allow us to compare $g^{P}$ to $g\left(a_{m e d}\right)$, and even to $g^{L}$ and $g^{*}$ when the comparison of $\hat{y}$ and $y_{\text {med }}$ is extended to $y_{M}$. The benchmark $g\left(a_{\text {med }}\right)$ is also interesting per se, because it constitutes the natural extension to our setting of the equilibrium identified by Alesina et al. (1999) (with lump sum financing).

We first study the case where the distributions of income and of location are independent from each other. We then lift this assumption in order to understand the role played by the correlation between types.

\subsection{Independent distributions}

We prove the following proposition in Appendix 3.

Proposition 7 Assume proportional income taxation, that individuals vote first over the public good's size and then over its type, and that the two individual traits $\beta$ and $y$ are independently distributed. If (i) the taste parameter $\beta$ is uniformly distributed and (ii) the distribution of income $y$ is symmetrical around $y_{M}$, then the equilibrium proportional public good level is the one most-preferred by the individual with the median income and the median distance to the median when faced with $p=\alpha_{\text {med }}$ (i.e., $g^{P}=g\left(a_{m e d}\right)$ ). If condition (i) holds together with $H_{y}$ convex (resp., concave), then $g\left(a_{\text {med }}\right) \leq g^{P}$ (resp., $\left.g\left(a_{\text {med }}\right) \geq g^{P}\right)$.

Observe first that the situation where the bivariate distribution $H$ is uniform (which is the one most of the literature has focused on, such as in Etro (2006), Gregorini (2009) and Perroni and Scharf (2001)) is a special case where assumptions (i) and (ii) in Proposition 7 are satisfied. We now show how Proposition 7 allows us to rank the various levels of public good reported in Table 2. Assumption (i) in Proposition 7 ensures that $\beta_{\text {med }}=\beta_{M}$ (and hence that $g^{L}=g^{*}$ ) while (ii) guarantees that $y_{\text {med }}=y_{M}$. Put together, we show in the appendix that they imply that $a_{1}=a_{\text {med }}$, and thus (see Table 2 ) that $\hat{y}=y_{\text {med }}$-i.e., that $g^{P}=g^{L}=g\left(a_{\text {med }}\right)=g^{*}$.

If condition (i) in Proposition 7 is satisfied while $H_{y}$ is convex, Proposition 7 states that $g\left(a_{\text {med }}\right) \leq g^{P}$ and we obtain that

$$
\frac{\widehat{y}}{\beta_{\text {med }} y_{M}}=V^{\prime}\left(a_{1} y_{M}\right) \leq V^{\prime}\left(a_{\text {med }} y_{M}\right)=\frac{y_{\text {med }}}{\beta_{\text {med }} y_{M}} \text {. }
$$


If we further make the empirically relevant assumption that $y_{m e d}<y_{M},{ }^{16}$ we obtain that

$$
V^{\prime}\left(a_{1} y_{M}\right) \leq V^{\prime}\left(a_{m e d} y_{M}\right)=\frac{y_{\text {med }}}{\beta_{\text {med }} y_{M}}<\frac{1}{\beta_{\text {med }}},
$$

i.e., that the public good is overprovided under our benchmark, and even more so with the sequential procedure with proportional financing $\left(g^{P} \geq g\left(a_{\text {med }}\right)>g^{*}=g^{L}\right) .{ }^{17}$

Proposition 7 has not exhausted the set of possible (and interesting) distributions of income. We now provide numerical illustrations of the comparison between $g^{L}, g^{P}$ and $g^{*}$ when the two individual traits are independently distributed, with $H_{\beta}$ uniform and $H_{y}$ a Beta distribution. Figure 6 (a) varies the skewness of the income distribution for the family of Beta distributions with linear frequencies (i.e., when either the first or the second parameter of the distribution is equal to 1 ). It reports the values of the proportional tax rates $a_{1}$, $a_{m e d}$ and also of $a^{*}$, defined as the rate that ensures the provision of the socially optimal amount of public good (i.e., $a^{*} y_{M}=g^{*}$ ). ${ }^{18}$ The first condition of Proposition 7 is satisfied by assumption, so that $g^{L}=g^{*}=a^{*} y_{M}$. A skewness of zero corresponds to the uniform distribution $(\operatorname{Beta}(1,1))$, for which, according to Proposition $7, g\left(a_{m e d}\right)=g^{P}$, so that the sequential procedure and our benchmark both correspond to the socially optimal public good level $\left(g^{P}=g\left(a_{m e d}\right)=g^{*}\right)$. A negative skewness within the family of linear frequencies means that the function $H_{y}$ is convex. Proposition 7 then shows that $g^{P} \geq g\left(a_{m e d}\right)$, but we are not able to compare them analytically to $g^{*}$ since we have that $y_{m e d}>y_{M}$. Figure 6 (a) shows that $a_{1}>a^{*}>a_{m e d}$ (i.e., $g^{P}>g^{*}>g\left(a_{m e d}\right)$ ) if the skewness of $H_{y}$ is very negative, and that $a^{*}>a_{1}>a_{m e d}$ (i.e., $g^{*}>g^{P}>g\left(a_{m e d}\right)$ ) if the skewness is negative but small in absolute value. In words, our benchmark leads to underprovision (compared with both the socially optimal level and the result of a sequential voting procedure) in all cases with negative skewness. Moreover, the sequential procedure overprovides public good (compared to the social optimum) if the skewness is very negative. When the skewness of $H_{y}$ is positive in Figure 6 (a), we obtain that $H_{y}$ is concave, and by Proposition 7 that $g^{P} \leq g\left(a_{m e d}\right)$. In that case, we obtain that $y_{m e d}<y_{M}$, so that we cannot compare analytically $g^{P}$ and $g\left(a_{m e d}\right)$ to $g^{*}$. We obtain numerically that $a^{*}<a_{1}<a_{\text {med }}$ (i.e., $g^{*}<g^{P}<g\left(a_{\text {med }}\right)$ ) in Figure 6 (a): both the sequential procedure and our benchmark lead to overprovision of the public good.

\footnotetext{
${ }^{16}$ Note that the assumption that $H_{y}$ is convex neither ensures nor precludes that $y_{m e d}<y_{M}$.

${ }^{17}$ In the case where assumption (i) in Proposition 7 is satisfied together with $H_{y}$ concave, proving that the public good is underprovided under proportional taxation would require the unreasonable assumption that $y_{\text {med }}>y_{M}$. However, if assumption (i) is dropped as well so that $\frac{y_{m e d}}{y_{M}}>\frac{\beta_{m e d}}{\beta_{M}}$, we obtain that $g^{P} \leq g\left(a_{\text {med }}\right)<g^{*}$.

${ }^{18}$ We have chosen to report tax rates in Figures 6 and 7 because the scale is easier to interpret. The text concentrates on public good levels, the link between comparison of tax rates and of public good levels being straightforward since $g=a y_{M}$ for all values of $a$.
} 
Figure 6 (b) performs the same exercise for another family of Beta income distribution functions, namely those with single-peaked and non-linear frequencies. ${ }^{19} \mathrm{~A}$ zero skewness corresponds to a symmetrical $h_{y}$, and thus by Proposition 7 to $g^{P}=g\left(a_{m e d}\right)=g^{*}$. Observe also that $H_{y}$ is neither convex nor concave, so that we cannot apply Proposition 7 when the skewness is not nil. We learn from Figure $6(\mathrm{~b})$ that $a_{\text {med }}<a_{1}<a^{*}$ (i.e., $g\left(a_{\text {med }}\right)<g^{P}<g^{*}$ ) when skewness is negative and that $a^{*}<a_{1}<a_{\text {med }}$ (i.e., $g\left(a_{m e d}\right)>g^{P}>g^{*}$ ) when skewness is positive. That is, both the sequential procedure with proportional taxation and our benchmark underprovide (resp., overprovide) public good when the skewness of the income distribution is negative (resp., positive).

In the next section, we go beyond the independent distributions assumption and study the impact of the correlation between income and location.

\subsection{Correlation between income and location}

When the two marginal distributions are correlated, the picture becomes more complex. Figure 7 shows both $a_{m e d}$ and $a_{1}$ as functions of the correlation between $\beta$ and $y$ when $H_{\beta}$ and $H_{y}$ are uniform and when the Gumbel copula is used (see Appendix 4 for details). With $H_{\beta}$ uniform, we have that $\beta_{\text {med }}=\beta_{M}$ so that $g^{L}=g^{*}$. With $H_{y}$ uniform, we obtain that $y_{M}=y_{m e d}$, so that we infer that $a^{*}=a_{m e d}$. In words, the uniformity of the two marginal distributions ensures that both the sequential procedure (over public good's size first and then type) with lump sum financing and our benchmark lead to socially optimal public good levels: $g^{L}=g\left(a_{m e d}\right)=g^{*}$. Moreover, in the case of independence between $H_{y}$ and $H_{\beta}$, we know from Proposition 7 that $g^{P}=g\left(a_{\text {med }}\right)$. Figure 7 shows that $a_{\text {med }}=a_{1}$ also when the correlation between income and position is perfect; a simple look at Figure 5 should convince the reader that this is so, since the median iso-tax line remains the same in both the cases of perfect correlation and of independence. We further learn from Figure 7 that $a_{m e d}>a_{1}$ when the correlation is strictly in between zero and one. This means that, except in the cases of zero or perfect correlation between income and taste, the sequential determination of the proportional tax rate $a$ first and then $p$ leads to systematic underprovision of public good in the case illustrated in Figure 7, while the other equilibria studied ensure the socially efficient public good level: $g^{P}<g^{L}=g\left(a_{m e d}\right)=g^{*}$.

\footnotetext{
${ }^{19}$ More precisely, we study $\operatorname{Beta}(c, d)$ distributions where one parameter is equal to 2 . Negatively skewed distributions correspond to $c>2$ and $d=2$ while positive skewness corresponds to $c=2$ and $d>2$. The case with zero skewness corresponds to $\operatorname{Beta}(2,2)$.
} 
Insert Figure 7 around here

\section{Conclusion}

In this paper, we study majority voting over the size and location of a public good. Individuals differ both in income and in their preferences for the public good location, so that the polity is summarized by a bivariate distribution of these two traits. Public expenditures are financed by affine income taxation: we consider both the case of a lump sum tax and of a proportional income tax. We study both the simultaneous and the sequential determinations of the public good size and location. We show that, while the choice of the type of public good follows the traditional median logic, the majoritarian determination of the taxation rate is more subtle and need not coincide with the preferences of a median income citizen. With lump sum financing, income heterogeneity plays no role and the sequential equilibrium consists of the median location together with the public good level most-preferred by the individual located at the median distance to the median (as in Alesina et al. (1999)). This policy bundle also constitutes an equilibrium with simultaneous voting in the special case of a median uniform bivariate distribution. With proportional taxation, there is no policy equilibrium with simultaneous voting even if the bivariate distribution is uniform. We offer a complete characterization of the equations describing the sequential equilibrium in the general case (no assumption on the bivariate distribution of traits). We show why and how our results depart from those obtained in a natural benchmark where the public good level chosen is the one most-preferred by the individual with the median distance to the median and the median income. We obtain a lower public good level than this benchmark when the income distribution is concave, such as with positively skewed Beta distributions, and when the correlation between income and location is positive but not perfect. We also compare majority voting allocations with the socially optimal one.

This paper is a first step towards a more general analysis of the majoritarian decision process when the policy set is multidimensional. Further research would shed additional light on the robustness of the conclusions established in the present paper. First, we would like to know to which extent these results remain valid for a broader class of settings and of utility functions. Is the quasi-linear form an inescapable constraint? The specific utility functions used in this paper are common in the nation formation literature which has motivated us, but we conjecture that some features of the equilibrium outcome can be extended to more general formulations. A second promising direction of research would consist in comparing 
the equilibrium reached under sequential voting with another popular solution due to Kramer (1972) and Shepsle (1979), in which separate committees vote on the various issues at hand. De Donder, Le Breton and Peluso (2011) contain some preliminary answers to these two questions. A third direction of research would consist in integrating the majority voting approach described in this paper to a more general game of nation formation.

\section{Appendix}

In the two following subsections, we investigate the conditions under which the indirect utility functions of the citizens in the lump sum case and in the proportional case are strictly quasi- concave. The developments are based on a straightforward application of the classical differential test of strict quasi-concavity as exposed for instance in Green, Mas-Colell and Whinston (1995)).

\subsection{Appendix 1}

In this first appendix, we demonstrate that, under some appropriate conditions, the (indirect) utility $U$ of a citizen of type $(\alpha, y)$ in the case of lump sum taxation

$$
U(p, b)=V(b)[\lambda-|\alpha-p|]+y-b
$$

is strictly quasi-concave. Without loss of generality, consider the case where $\alpha>p$. The bordered Hessian matrix $D^{2} U(p, b)$ attached to $U$ is here

$$
\left(\begin{array}{ccc}
V^{\prime \prime}(b)(\lambda-\alpha+p) & V^{\prime}(b) & V^{\prime}(b)(\lambda-\alpha+p)-1 \\
V^{\prime}(b) & 0 & V(b) \\
V^{\prime}(b)(\lambda-\alpha+p)-1 & V(b) & 0
\end{array}\right)
$$

The utility function is strictly quasi-concave if and only if the determinant of this matrix is positive -i.e., if

$$
2\left(V^{\prime}(b)(\lambda-\alpha+p)-1\right) V(b) V^{\prime}(b)-(V(b))^{2} V^{\prime \prime}(b)(\lambda-\alpha+p)>0,
$$

which is equivalent to the inequality

$$
2\left(V^{\prime}(b)\right)^{2}-V(b) V^{\prime \prime}(b)>\frac{2 V^{\prime}(b)}{\lambda-\alpha+p} .
$$

The right hand side is decreasing in $p$. Therefore, it is enough to check this inequality for $p=0$ - i.e.,

$$
\frac{2\left(V^{\prime}(b)\right)^{2}-V(b) V^{\prime \prime}(b)}{2 V^{\prime}(b)}>\frac{1}{\lambda-\alpha} .
$$


The relevant (i.e., Pareto) range of values of $b$ is the interval $\left[V^{\prime-1}\left(\frac{1}{\lambda-1}\right), V^{\prime-1}\left(\frac{1}{\lambda}\right)\right]$. The above inequality is tighter to satisfy when $\alpha=1$ and $V^{\prime}(b)$ is small. Therefore, it will hold true for all $b \in\left[V^{\prime-1}\left(\frac{1}{\lambda-1}\right), V^{\prime-1}\left(\frac{1}{\lambda}\right)\right]$ and all $\alpha \in[0,1]$ if

$$
\frac{1}{\lambda}-\frac{V(b) V^{\prime \prime}(b)}{2 V^{\prime}(b)}>\frac{1}{\lambda-1},
$$

i.e. if

$$
-\frac{V(b) V^{\prime \prime}(b)}{2 V^{\prime}(b)}>\frac{1}{\lambda(\lambda-1)},
$$

which holds true when $V$ is concave enough or/and $\lambda$ is large enough. For the sake of illustration, consider the isoelastic $\operatorname{case}^{20} V(b)=b^{\delta}$ with $\left.\delta \in\right] 0,1[$. The above condition simplifies to

$$
\frac{(1-\delta)}{2} b^{\delta-1}>\frac{1}{\lambda(\lambda-1)}
$$

Since $b^{\delta-1} \geq \frac{1}{\lambda \delta}$, the inequality will hold true if

$$
\frac{(1-\delta)}{2 \delta}>\frac{1}{\lambda-1}
$$

or equivalently if

$$
\delta<\frac{\lambda-1}{\lambda+1}
$$

\subsection{Appendix 2}

In this second appendix, we prove that under appropriate conditions, the (indirect) utility $W$ of a citizen of type $(\alpha, y)$ in the case of proportional taxation,

$$
\left.W(p, a)=V\left(a y_{M}\right)\right)[\lambda-|\alpha-p|]+(1-a) y,
$$

is strictly quasi-concave. Without loss of generality, we consider the case where $\alpha>p$. The bordered Hessian matrix $D^{2} W(p, a)$ attached to $W$ is here

$$
\left(\begin{array}{ccc}
V^{\prime \prime}\left(a y_{M}\right)\left(y_{M}\right)^{2}(\lambda-\alpha+p) & V^{\prime}\left(a y_{M}\right) y_{M} & V^{\prime}\left(a y_{M}\right) y_{M}(\lambda-\alpha+p)-y \\
V^{\prime}\left(a y_{M}\right) y_{M} & 0 & V\left(a y_{M}\right) \\
V^{\prime}\left(a y_{M}\right) y_{M}(\lambda-\alpha+p)-y & V\left(a y_{M}\right) & 0
\end{array}\right)
$$

The utility function is strictly quasi-concave if and only if the determinant of this matrix is positive -i.e., if

$$
2\left(V^{\prime}\left(a y_{M}\right) y_{M}(\lambda-\alpha+p)-y\right) V\left(a y_{M}\right) V^{\prime}\left(a y_{M}\right) y_{M}-\left(V\left(a y_{M}\right)\right)^{2} V^{\prime \prime}\left(a y_{M}\right)\left(y_{M}\right)^{2}(\lambda-\alpha+p)>0,
$$

\footnotetext{
${ }^{20}$ In the isoelastic case, the sufficient condition can be improved to the condition $\frac{(1+\delta)}{2 \delta}>\frac{\lambda}{\lambda-1}$ or even to the condition $\frac{(1+\delta)}{2 \delta}>\frac{\lambda}{\lambda-\alpha}$ if we dont look for a condition uniform with respect to $\alpha$.
} 
which is equivalent to

$$
2\left(V^{\prime}\left(a y_{M}\right) y_{M}(\lambda-\alpha+p)-y\right) V^{\prime}\left(a y_{M}\right)-V\left(a y_{M}\right) V^{\prime \prime}\left(a y_{M}\right) y_{M}(\lambda-\alpha+p)>0,
$$

and, after some rearrangements, to

$$
y_{M}\left[2\left(V^{\prime}\left(a y_{M}\right)\right)^{2}-V\left(a y_{M}\right) V^{\prime \prime}\left(a y_{M}\right)\right]>\frac{2 V^{\prime}\left(a y_{M}\right) y}{\lambda-\alpha+p} .
$$

As in Appendix 1, the right hand side is decreasing in $p$. Therefore, it is enough to check this inequality for $p=0$ - i.e. that

$$
\frac{y_{M}}{y} \frac{2\left(V^{\prime}\left(a y_{M}\right)\right)^{2}-V\left(a y_{M}\right) V^{\prime \prime}\left(a y_{M}\right)}{2 V^{\prime}\left(a y_{M}\right)}>\frac{1}{\lambda-\alpha},
$$

or equivalently that

$$
\frac{y_{M}}{y} V^{\prime}\left(a y_{M}\right)-\frac{y_{M}}{y} \frac{V\left(a y_{M}\right) V^{\prime \prime}\left(a y_{M}\right)}{2 V^{\prime}\left(a y_{M}\right)}>\frac{1}{\lambda-\alpha} .
$$

The relevant range of values of $a y_{M}$ is the interval $\left[V^{\prime-1}\left(\frac{\bar{y}}{(\lambda-1) y_{M}}\right), V^{\prime-1}\left(\frac{\bar{y}}{\lambda y_{M}}\right)\right]$. The above inequality is tighter to satisfy when $\alpha=1$ and $V^{\prime}\left(a y_{M}\right)$ is small. Therefore, it will hold true for all $a y_{M} \in\left[V^{\prime-1}\left(\frac{\bar{y}}{(\lambda-1) y_{M}}\right), V^{\prime-1}\left(\frac{\bar{y}}{\lambda y_{M}}\right)\right]$ and all $\alpha \in[0,1]$ if

$$
-\frac{y_{M}}{y} \frac{V\left(a y_{M}\right) V^{\prime \prime}\left(a y_{M}\right)}{2 V^{\prime}\left(a y_{M}\right)}>\frac{1}{\lambda(\lambda-1)},
$$

which, as in the lump sum case, holds true when $V$ is concave enough or/and $\lambda$ is large enough. In the isoelastic case $V\left(a y_{M}\right)=\left(a y_{M}\right)^{\delta}$ with $\left.\delta \in\right] 0,1\left[\right.$, and since $\left(a y_{M}\right)^{\delta-1} y_{M} \geq$ $\frac{y}{y_{M}} \frac{1}{\lambda \delta}$, the inequality will hold true if

$$
\frac{(1-\delta)}{2 \delta}>\frac{1}{\lambda-1}
$$

as in appendix 1.

\subsection{Appendix 3: Proof of Proposition 7}

With independent distributions of $\beta$ and $y$, we have $h(\beta, y)=h_{\beta}(\beta) h_{y}(y)$ and $a_{m e d}$ is solution to the equation

$$
\int_{\eta}^{\lambda} h_{\beta}(\beta) H_{y}\left(\beta \varphi\left(a_{1}\right)\right) d \beta=\frac{1}{2} .
$$


Under assumption (i), $H_{\beta}(\beta)$ is the uniform distribution over $[\eta, \lambda]$ and $a_{1}$ solves

$$
\int_{\eta}^{\lambda} H_{y}(\beta \varphi(a)) d \beta=\frac{\lambda-\eta}{2}
$$

while $a_{m e d}$ is such that

$$
H_{y}\left(\frac{\lambda+\eta}{2} \varphi\left(a_{m e d}\right)\right)=\frac{1}{2}
$$

The two tax rates do not coincide in general. It is of interest to identify the properties of $H_{y}$ which would lead to $a_{1}$ being smaller than, equal to, or larger than the benchmark $a_{m e d}$.

We have that, if $H_{y}$ is concave (respectively, convex), then

$$
\int_{\eta}^{\lambda} H_{y}(\beta \varphi(a)) d \beta \leq(\text { respectively, } \geq) H_{y}\left(\frac{\lambda+\eta}{2} \varphi(a)\right) .
$$

Since

$$
\varphi^{\prime}(a) \equiv y_{M}^{2} V^{\prime \prime}(g(a))<0,
$$

and $H_{y}$ is increasing, we have proved the last sentence of the statement of Proposition 7.

The case where $H_{y}$ has concave and convex sections is also important as reflected by the standard assumption of single-peaked density. If we assume that $h_{y}$ is symmetrical around $y_{M}=\bar{y} / 2$, we obtain that $a_{m e d}$ is given by

$$
\frac{\lambda+\eta}{2} \varphi\left(a_{m e d}\right)=\frac{\bar{y}}{2}=y_{M}
$$

Replacing $a$ by $a_{m e d}$ in (24), and using the symmetry of $H_{y}$, we obtain

$$
\begin{aligned}
\int_{\eta}^{\lambda} H_{y}\left(\frac{\beta}{\beta_{M}} y_{M}\right) d \beta & =\int_{\eta}^{\frac{\lambda+\eta}{2}} H_{y}\left(\frac{\beta}{\beta_{M}} y_{M}\right) d \beta+\int_{\eta}^{\frac{\lambda+\eta}{2}}\left[1-H_{y}\left(\frac{\beta}{\beta_{M}} y_{M}\right)\right] d \beta \\
& =\int_{\eta}^{\frac{\lambda+\eta}{2}} d \beta=\frac{\lambda-\eta}{2}
\end{aligned}
$$

i.e., we have proved that $a_{m e d}=a_{1}$.

\subsection{Appendix 4: Gumbel copula and the correlation between in- come and location}

To assess the intrinsic role of independence, it is useful to introduce a measure of departure from independence. From Sklar's theorem (Sklar (1959)), there exists a joint distribution $C$ on $[0,1]^{2}$, called a copula, such that its two marginals are uniform on $[0,1]$ and

$$
H(\beta, y)=C\left(H_{\beta}(\beta), H_{y}(y)\right)
$$


As an illustration, we now assume that both $H_{\beta}$ and $H_{y}$ are uniformly distributed, and that the distribution $H$ is obtained using the Archimedean copula

$$
H(\beta, y)=\Phi^{-1}\left(\Phi\left(H_{\beta}(\beta)\right)+\Phi\left(H_{y}(y)\right)\right)
$$

where $\Phi$ is called the generator function. We provide an example with the so-called Gumbel copula, where the generator function is given by

$$
\Phi(x)=(\ln (x))^{-c}
$$

Increasing $c$ results in an increase in the correlation between $\beta$ and $y$ : the correlation is equal to zero (independence) when $c=0$, and increases to one as $c$ becomes large enough.

\section{References}

Alesina, A., Baqir, R. and W. Easterly. (1999), Public Goods and Ethnic Divisions, Quarterly Journal of Economics, 114, 1243-1284.

Alesina, A., Baqir, R. and C. Hoxby. (2004), Political Jurisdictions in Heterogeneous Communities, Journal of Political Economy, 112, 348-396.

Alesina, A. and E. Spolaore. (1997), On the Number and Sizes of Nations", Quarterly Journal of Economics, 112, 1027-1056.

Alesina, A. and E. Spolaore. (2003), The Size of Nations, The MIT Press, Cambridge.

Banks, J. and D. Austen-Smith (1999), Positive Political Theory : Collective Preference, Vol 1, Michigan University Press, Ann-Arbor.

Bolton, P. and G. Roland (1997), The Break-Up of Nations : A Political Economy Analysis, Quarterly Journal of Economics, 112, 1057-1090.

De Donder, P., Le Breton, M. and E. Peluso (2011) Majority Voting in Multidimensional Policy Spaces : Kramer-Shepsle versus Stackelberg, Journal of Public Economic Theory, forthcoming. Available as CEPR Discussion Paper 7646.

Drèze, J., Le Breton, M., Savvateev, A. and S. Weber (2008), Almost Subsidy-Free Spatial Pricing in a Multi-dimensional Setting, Journal of Economic Theory, 143(1), 275-291.

Etro, F (2006), Political Geography, Public Choice, 16, 517-535.

Gans, J. and M. Smart (1996), Majority Voting with Single-Crossing Preferences, Journal of Public Economics, 59, 219-238.

Green, J.R., Mas-Colell, A. and M.D. Whinston (1995), Microeconomic Theory, Oxford University Press, New York. 
Gregorini, F. (2009), Political geography and income inequalities, Working Paper 152 Dipartimento di Ecomomia Politica, Università di Milano - Bicocca, Febbraio 2009.

Haimanko, O., Le Breton, M. and S. Weber. (2005), Transfers in a Polarized Country : Bridging the Gap between Efficiency and Stability, Journal of Public Economics, 89, 12771303.

Jehiel, P. and S. Scotchmer (1997), Free Mobility and the Optimal Number of Jurisdictions, Annales d'Economie et de Statistique, 45, 219-231.

Jehiel, P. and S. Scotchmer (2001), Constitutional Rules of Exclusion in Jurisdiction Formation, Review of Economic Studies, 68, 393-413.

Kramer, G.H. (1972), Sophisticated Voting over Multidimensional Choice Spaces, Journal of Mathematical Sociology, 2, 165-180.

Le Breton, M. and S. Weber. (2003), The Art of Making Everybody Happy : How to Prevent a secession ?, IMF Staff Papers, 50, 403-435.

McKelvey, R., Ordeshook, P. and P. Ungar. (1980), Conditions for Voting Equilibria in Continuous Voter Distributions, Siam Journal of Applied Mathematics, 39, 161-168.

Ordeshook, P. (1986), Game Theory and Political Theory, Cambridge University Press, Cambridge.

Perroni, C. and K. Scharf (2001), Tiebout with Politics : Capital Tax Competition and Constitutional Choices, Review of Economic Studies, 68, 133-154.

Rothstein, P. (1990), Order Restricted Preferences and Majority Rule, Social Choice and Welfare, 7, 331-342.

Shepsle, K.A. (1979), Institutional Arrangements and Equilibrium in Multidimensional Voting Models, American Journal of Political Science, 23, 27-59

Sklar, A. (1959), Fonctions de Répartition à n Dimensions et leurs Marges, Publications de l'Institut de Statistique de l'Université de Paris, 8, 229-231. 
Table 1: Survey of the nation formation literature

\begin{tabular}{|c|c|c|c|c|c|c|c|c|c|}
\hline & \multicolumn{3}{|c|}{ Public good's choice } & \multicolumn{3}{|c|}{ Voters' heterogeneity } & \multirow{2}{*}{$\begin{array}{l}\text { Uniform } \\
\text { Distribution } \\
\text { of traits }\end{array}$} & \multicolumn{2}{|c|}{ Taxation } \\
\hline & Horizontal & Vertical & Both & Preferences & Income & Both & & Lump sum & Proportional \\
\hline Alesina Spolaore (1997) & $x$ & & & $x$ & & & $x$ & $x$ & \\
\hline Le Breton Weber (2003) & $\mathrm{x}$ & & & $\mathrm{x}$ & & & & $x$ & \\
\hline Bolton Roland (1997) & & $\mathrm{x}$ & & & $x$ & & & & $\mathrm{x}$ \\
\hline Jehiel Scotchmer (1997) & & $x$ & & $\mathrm{x}$ & & & $x$ & $x$ & \\
\hline Jehiel Scotchmer (2001) & & $x$ & & $x$ & & & $x$ & $x$ & \\
\hline Alesina, Baqir and Easterly (1999) & & & $x$ & $x$ & & & & $x$ & \\
\hline Alesina, Baquir and Hoxby (2004) & $\mathrm{x}$ & & & $\mathrm{X}^{1}$ & & & $x$ & $\mathrm{x}$ & \\
\hline Perroni Scharf (2001) & & & $\mathrm{x}$ & $\mathrm{x}$ & & & $x$ & $\mathrm{x}$ & \\
\hline Etro (2006) & & & $\mathrm{x}$ & $\mathrm{x}$ & & & $\mathrm{x}$ & $\mathrm{x}$ & \\
\hline Gregorini (2009) & & & $x$ & & & $X$ & $X$ & & $x$ \\
\hline
\end{tabular}

${ }^{1}$ Individuals differ in several dimensions (income, race, ethnicity, religion) but all these are summarized in a unidimensional index of distance, as in models of horizontal choices with heterogeneity in preferences. 
Table 2: Summary of results

\begin{tabular}{|l|l|l|}
\hline & Type $p$ & Level $g$ \\
\hline Optimal & $\alpha_{\text {med }}$ & $V^{\prime}\left(g^{*}\right)=\frac{1}{\beta_{M}}$ \\
\hline Sequential-Lump sum & $\alpha_{\text {med }}$ & $V^{\prime}\left(g^{L}\right)=\frac{1}{\beta_{\text {med }}}$ \\
\hline Sequential-Proportional & $\alpha_{\text {med }}$ & $g^{P}=a_{1} y_{M}$ with $^{1} V^{\prime}\left(g^{P}\right)=\frac{1}{\beta_{\text {med }}} \frac{\hat{y}}{y_{M}}$ for some $\hat{y}$ \\
\hline Benchmark & $\alpha_{\text {med }}$ & $g\left(a_{\text {med }}\right)=a_{\text {med }} y_{M}$ with $V^{\prime}\left(g\left(a_{m e d}\right)\right)=\frac{y_{m e d}}{\beta_{\text {med }} y_{M}}$ \\
\hline
\end{tabular}

${ }^{1}$ The proportional tax rate $a_{1}$ is implicitly defined by equation (21) while the income level $\hat{y}$ is implicitly defined by (18). 
Figure 1: Preference contour sets in (p, b) space with lump sum taxation
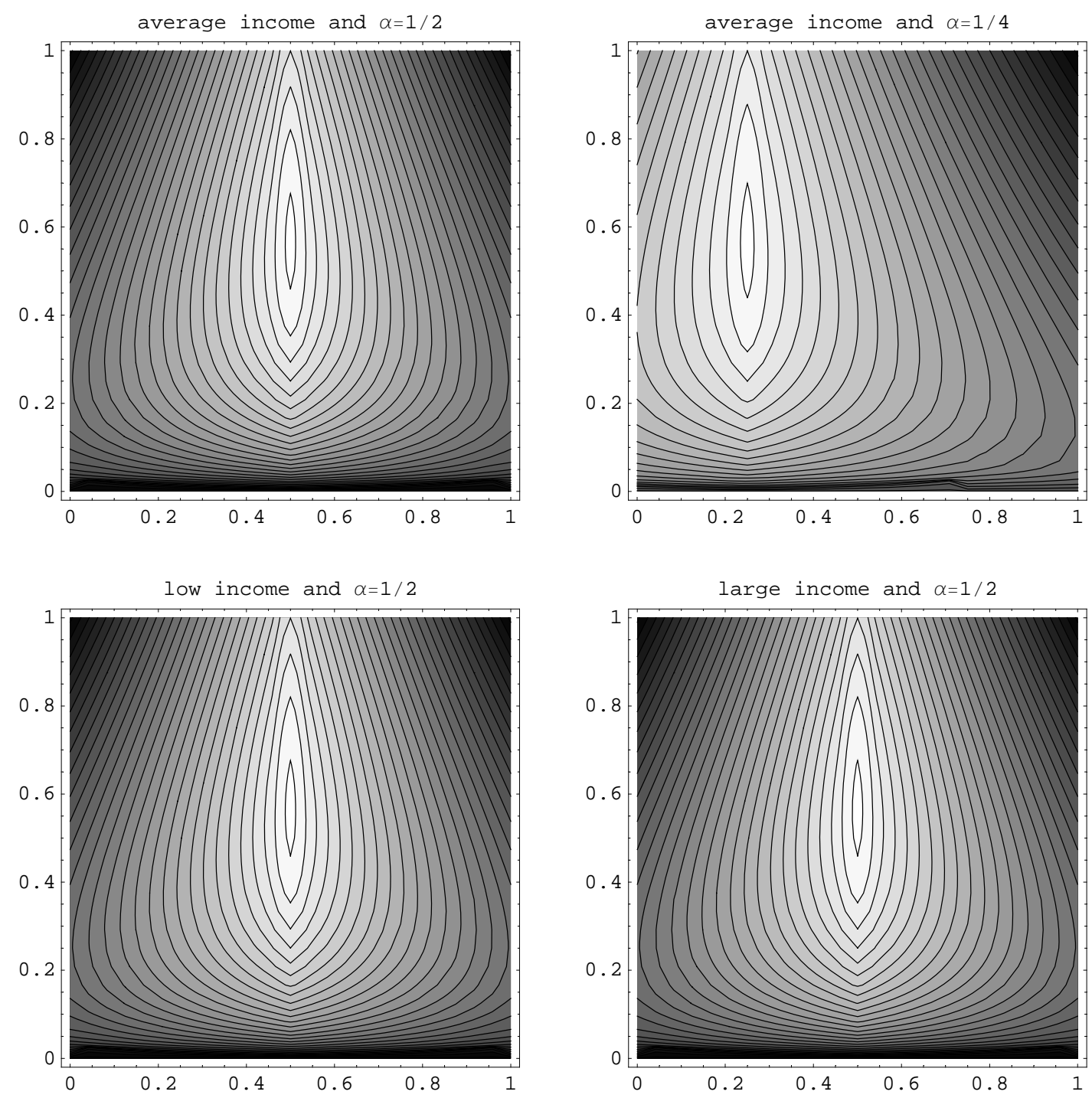
Figure 2: Iso-tax curves with lump-sum taxation

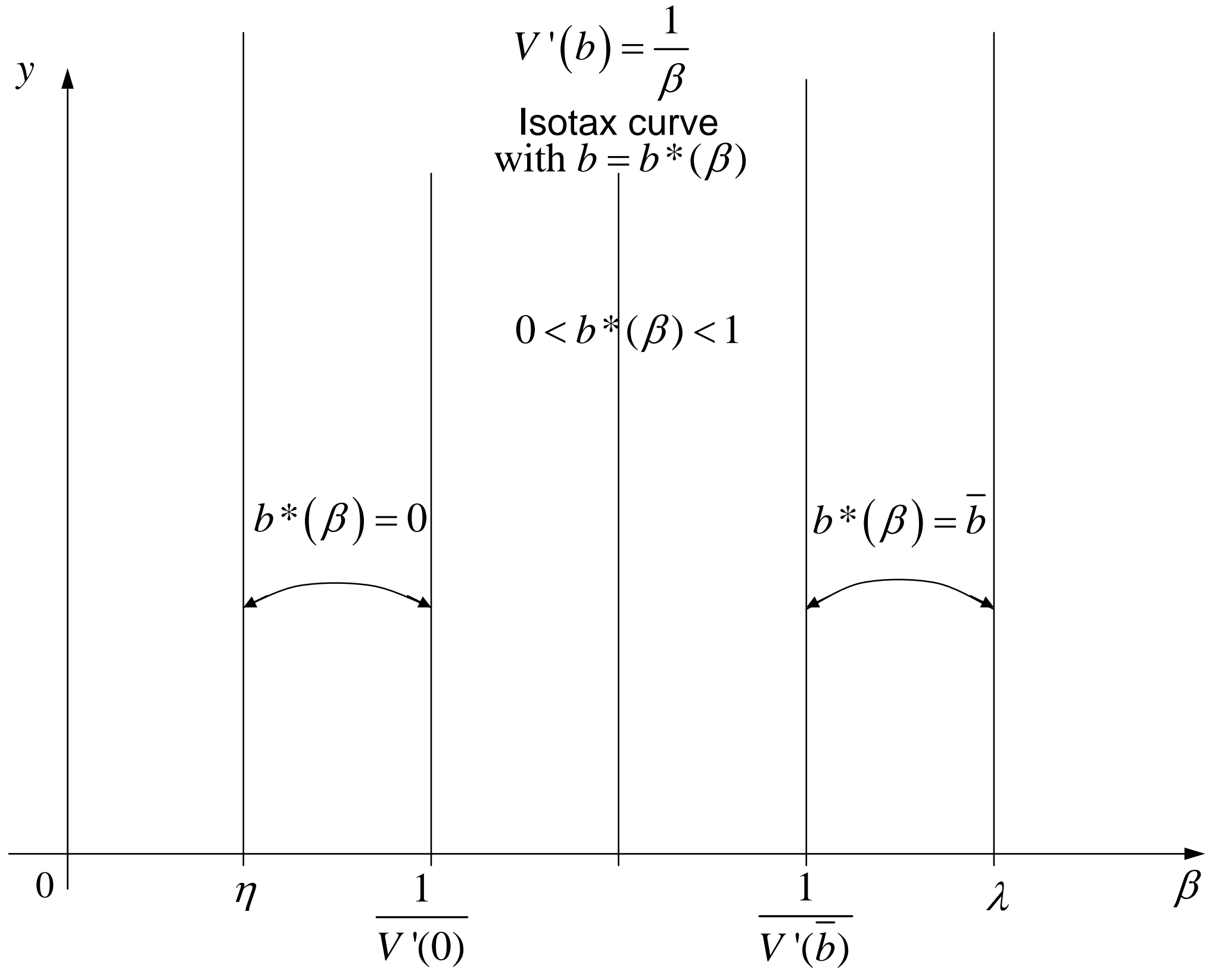


Figure 3 : Preference contour sets in (p, a) space with proportional taxation
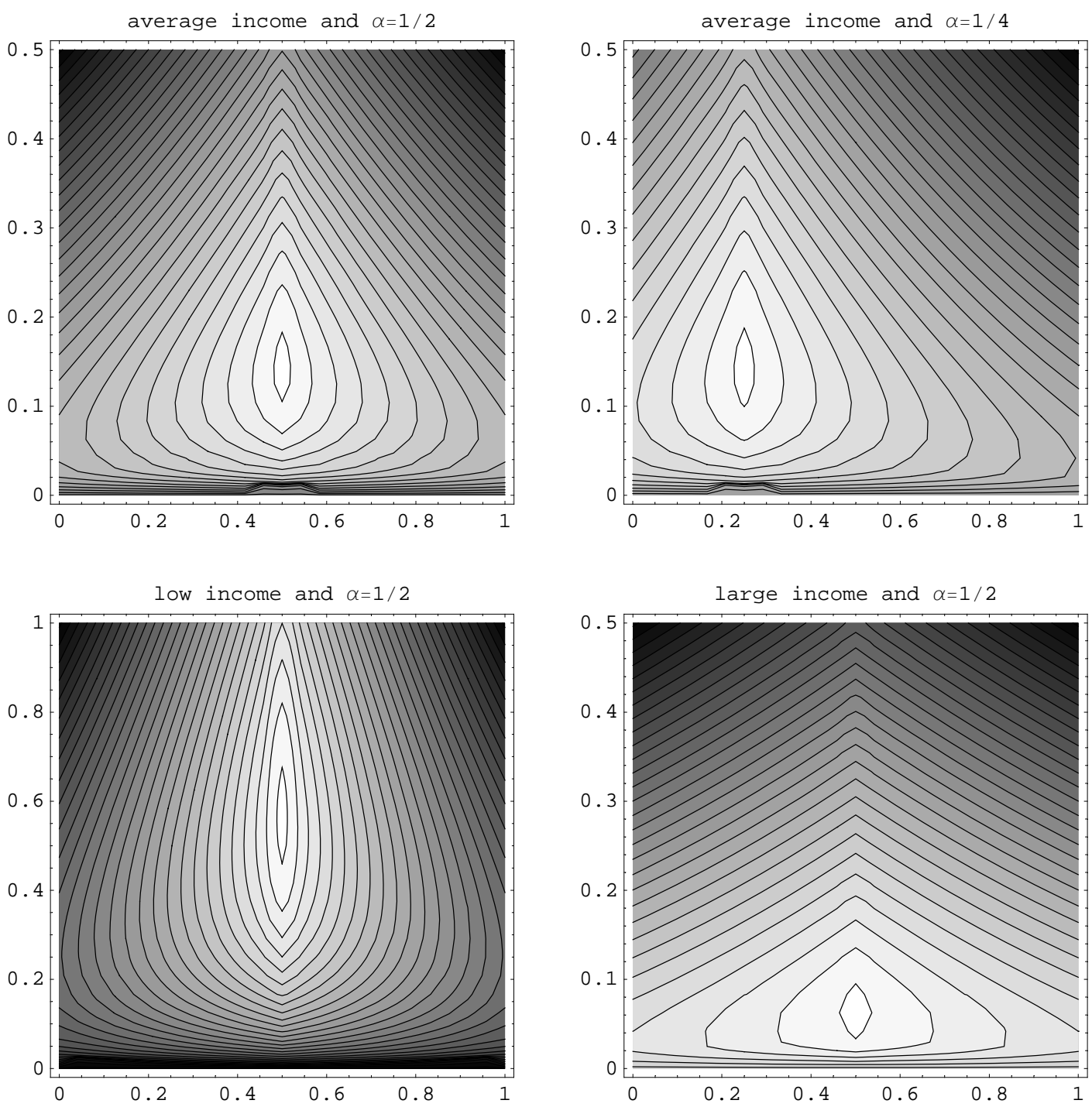
Figure 4: Set of individuals favoring a move in direction $d$, starting from $\left(\alpha_{\text {med }}, a\right)$

(a) Direction

$\left(d_{p}=0, d_{a}>0\right)$

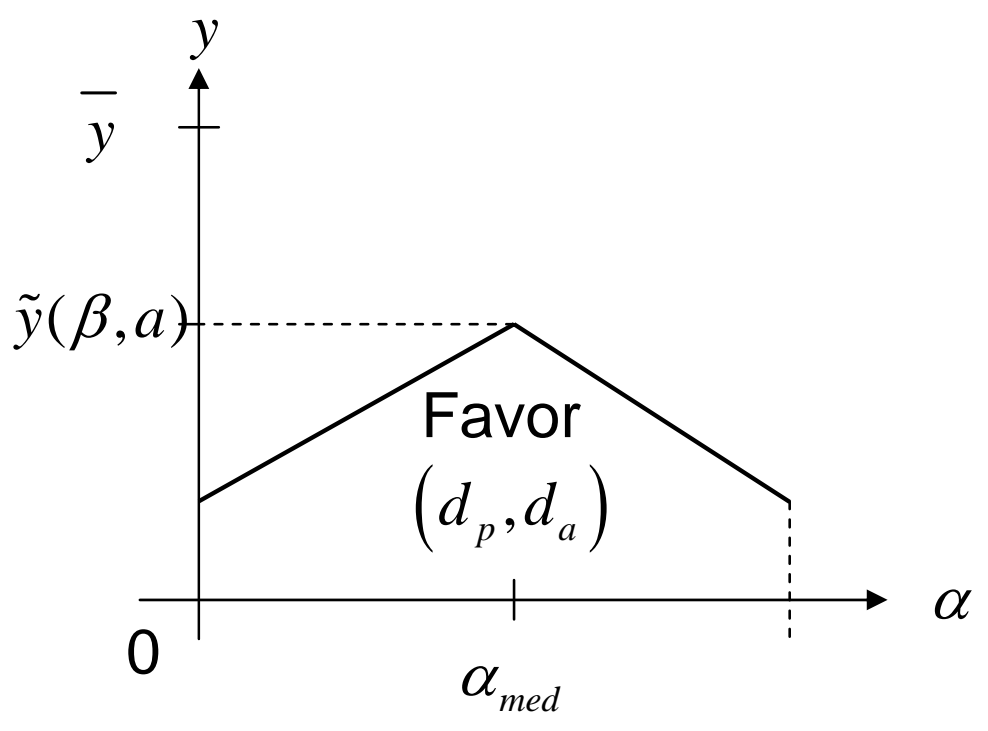

(b) Direction

$\left(d_{p}>0, d_{a}>0\right)$

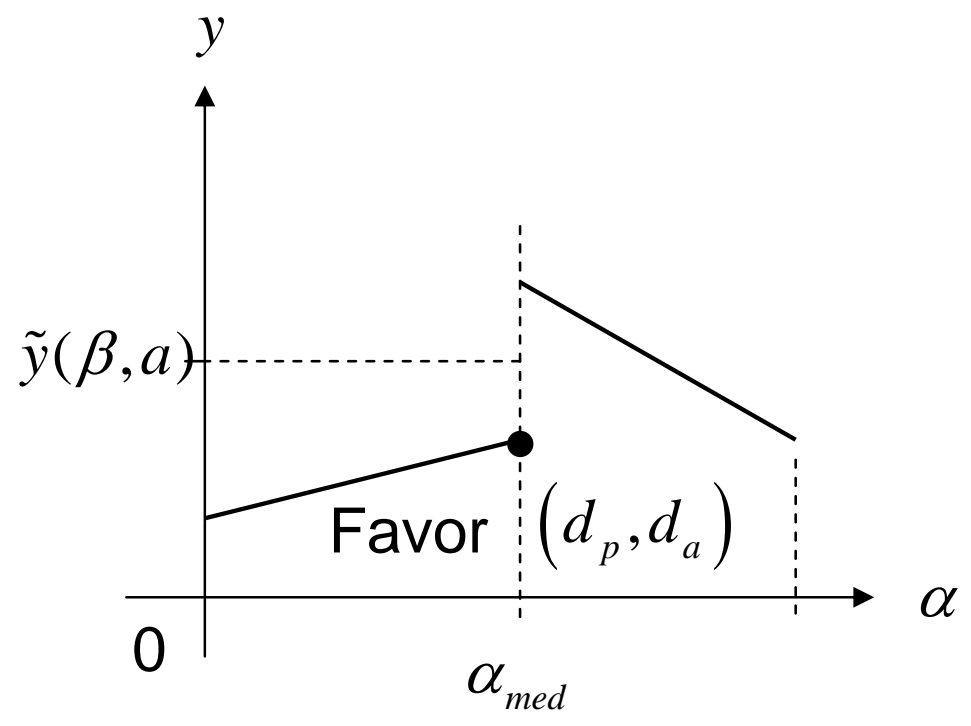

(c) Direction

$$
\left(d_{p}<0, d_{a}<0\right)
$$

with $d_{p}$ large enough $\bar{y} / 2$

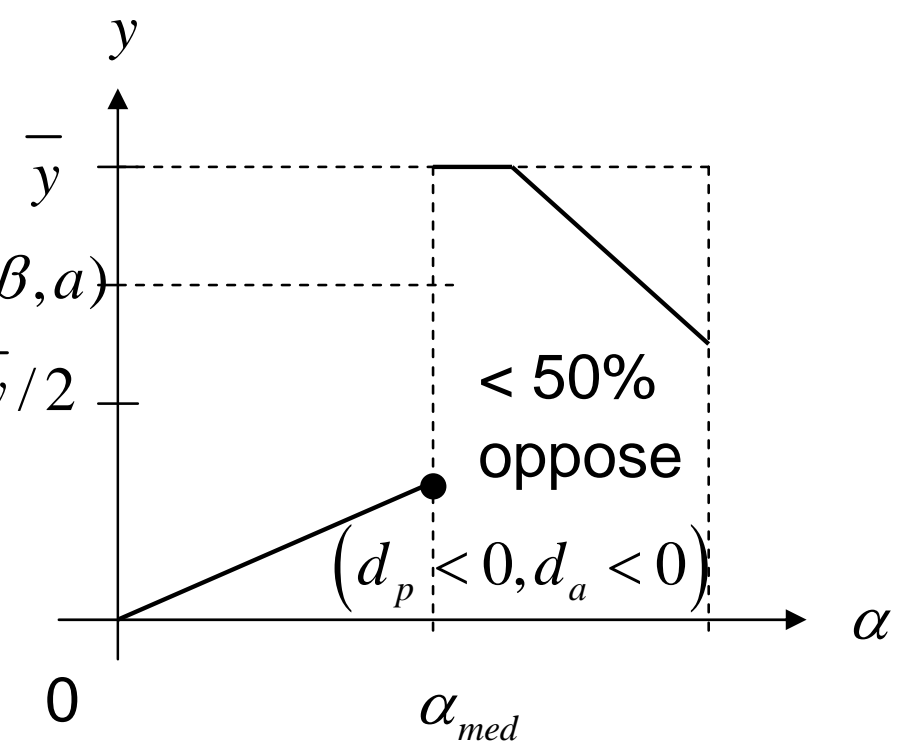


Figure 5: Iso-tax curves with proportional taxation

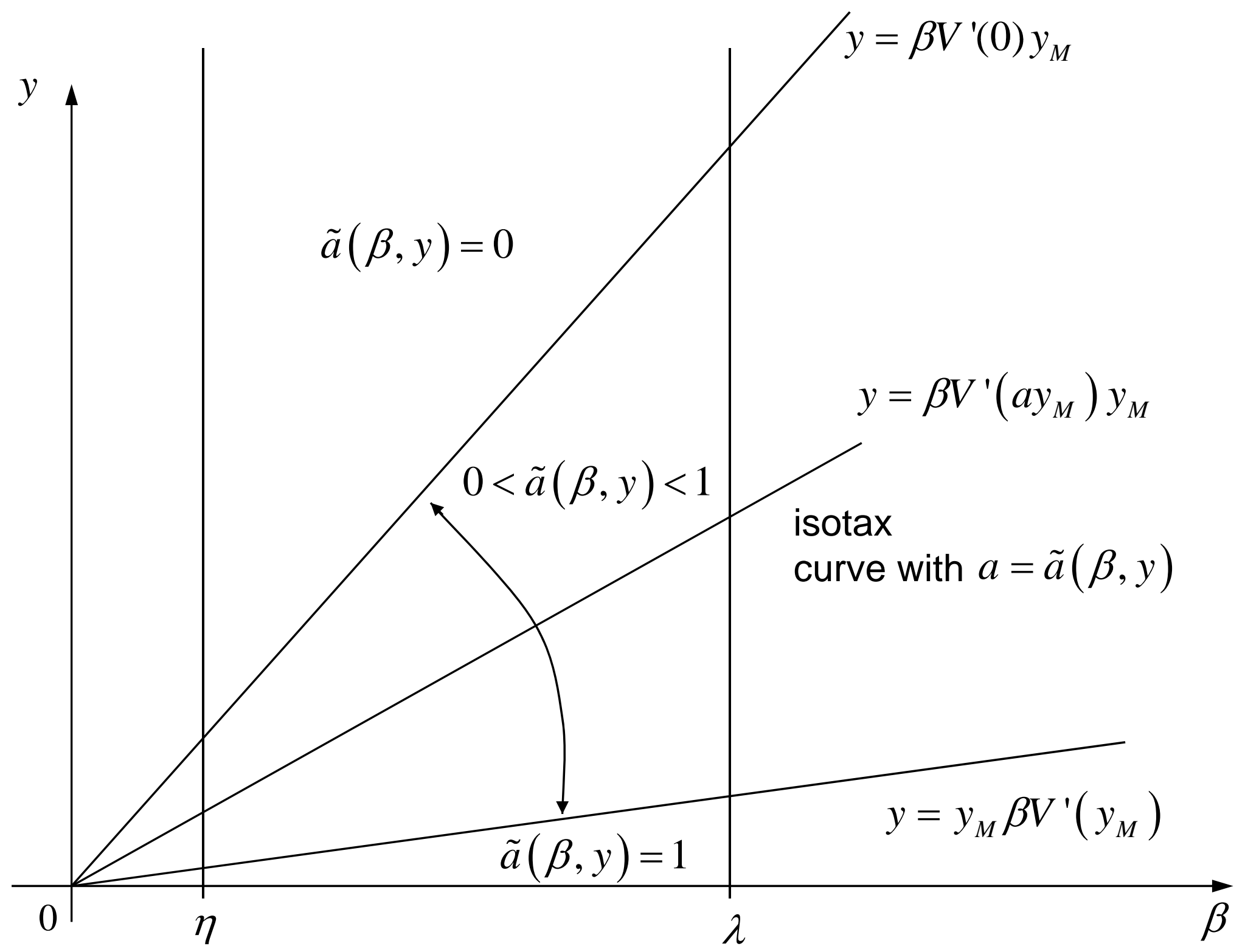


Figure 6 : Equilibrium tax rates as a function of skewness for Beta distributions

Panel (a) : Beta income distributions with linear frequency
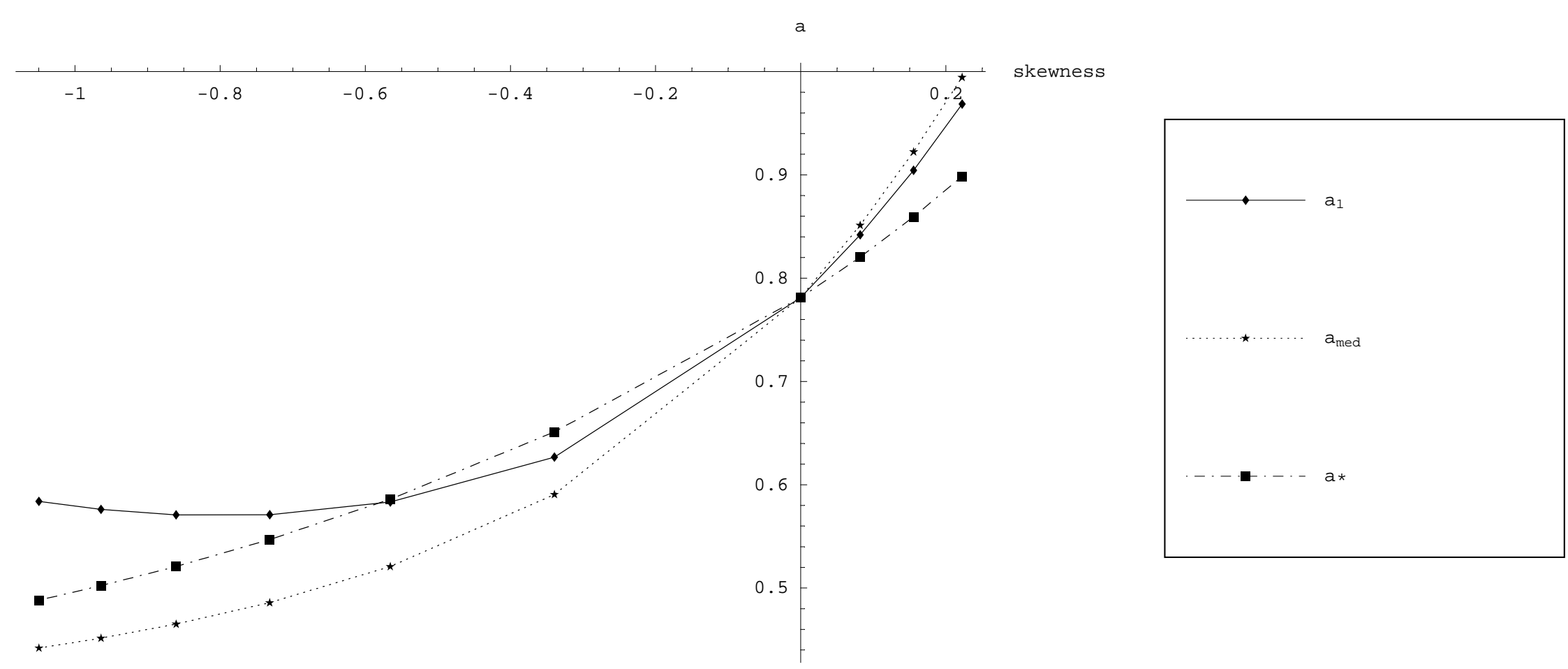
Panel (b) : Beta income distributions with single - peaaked non - linear frequency
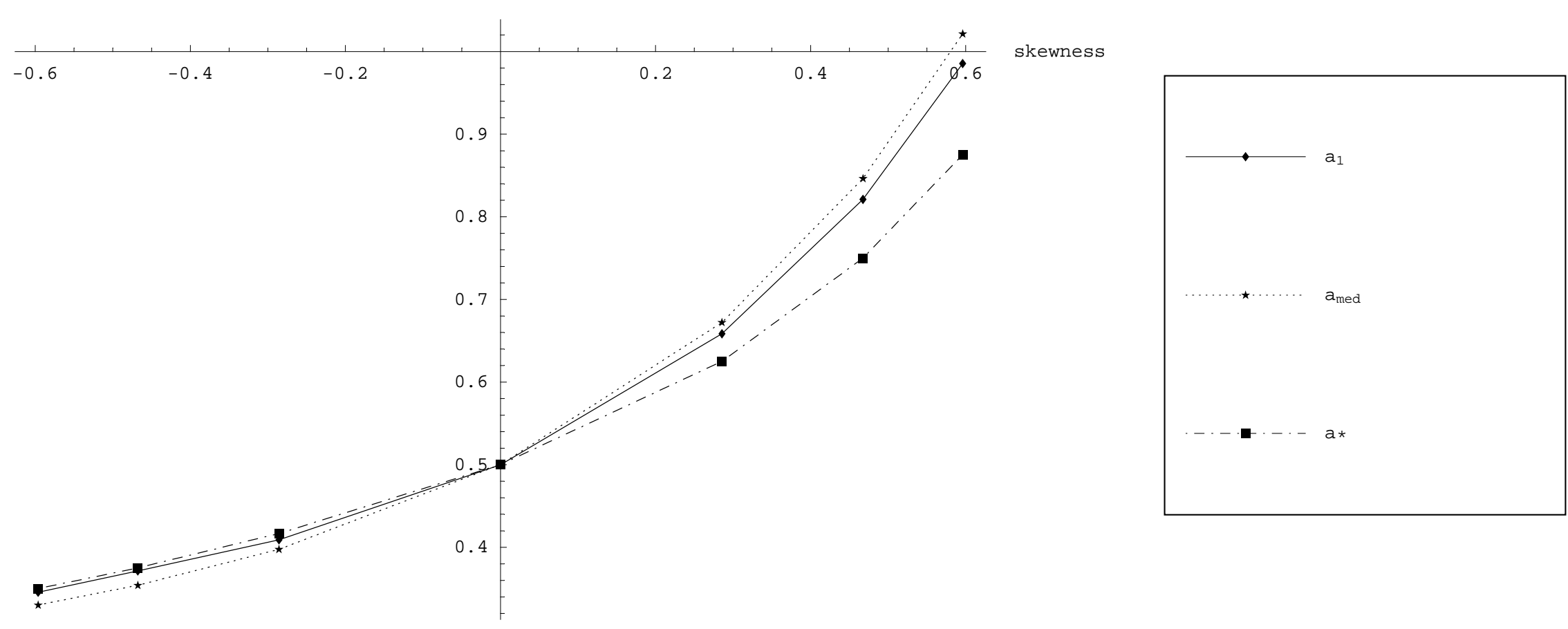
Figure 7 : Gumbel copula with $H_{\beta}$ and $H_{y}$ uniform

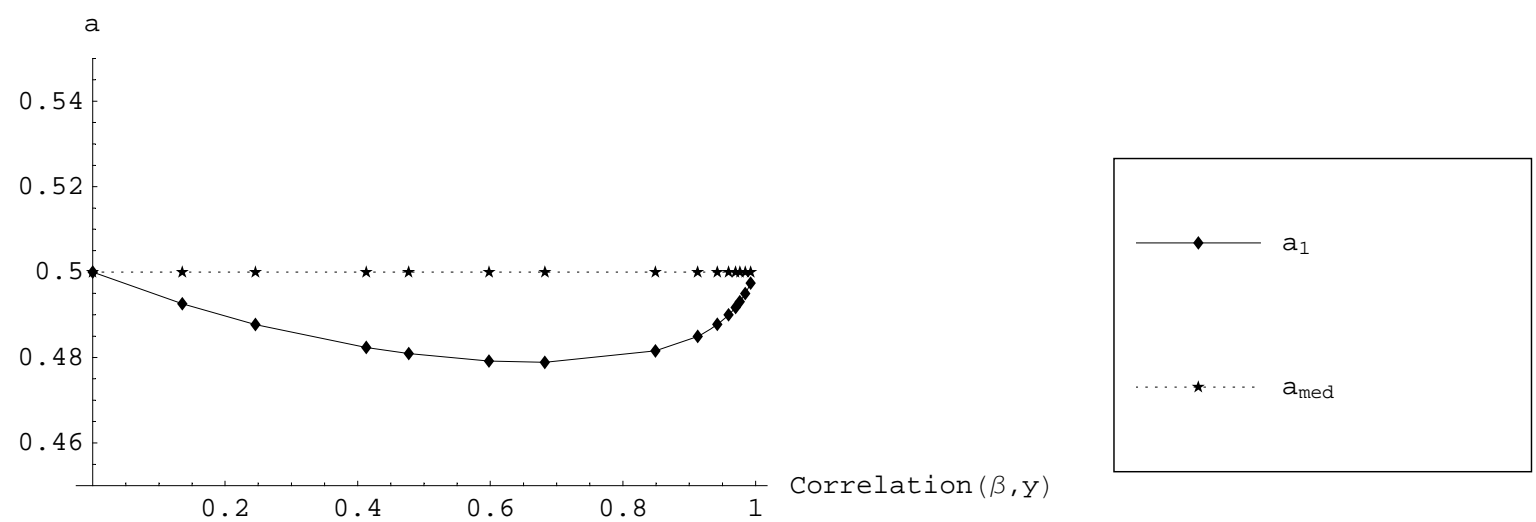

